\title{
Variability of Motor Neuron Spike Timing Maintains and Shapes Contractions of the Accessory Radula Closer Muscle of Aplysia
}

\author{
Yuriy Zhurov and Vladimir Brezina \\ Fishberg Department of Neuroscience, Mount Sinai School of Medicine, New York, New York 10029
}

\begin{abstract}
The accessory radula closer (ARC) muscle of Aplysia has long been studied as a typical "slow" muscle, one that would be assumed to respond only to the overall, integrated spike rate of its motor neurons, B15 and B16. The precise timing of the individual spikes should not much matter. However, but real B15 and B16 spike patterns recorded in vivo show great variability that extends down to the timing of individual spikes. By replaying these real as well as artificially constructed spike patterns into ARC muscles in vitro, we examined the consequences of this spike-level variability for contraction. Replaying the same pattern several times reproduces precisely the same contraction shape: the B15/B16 -ARC neuromuscular transform is deterministic. However, varying the timing of the spikes produces very different contraction shapes and amplitudes. The transform in fact operates at an interface between "fast" and "slow" regimens. It is fast enough that the timing of individual spikes greatly influences the detailed contraction shape. At the same time, slow integration of the spike pattern through the nonlinear transform allows the variable spike timing to determine also the overall contraction amplitude. Indeed, the variability appears to be necessary to maintain the contraction amplitude at a robust level. This phenomenon is tuned by neuromodulators that tune the speed and nonlinearity of the transform. Thus, the variable timing of individual spikes does matter, in at least two, functionally significant ways, in this "slow" neuromuscular system.
\end{abstract}

Key words: spike timing; neural code; neuromodulators; neuromuscular system; motor control; feeding behavior

\section{Introduction}

What constitutes the neural code (what features of a neuronal spike train carry functionally meaningful information) is still not clear in most instances. Is it simply the overall spike rate, or does the timing of the individual spikes carry additional information (König et al., 1996; Eggermont, 1998; deCharms and Zador, 2000)? Such questions have been studied particularly in sensory systems for the encoding of sensory information into the spike trains of sensory neurons and interneurons. However, analogous questions arise in motor systems for the control by motor neuron spike trains of muscle contractions.

Aplysia consummatory feeding behavior (biting, swallowing, and rejection of unsuitable food) is a cyclical behavior produced by the contractions of numerous muscles in the animal's feeding organ, the buccal mass, each controlled by the firing of its individual motor neurons, all driven ultimately by feeding motor programs generated by a central pattern generator (CPG) in the buccal ganglia (Kupfermann, 1974; Elliott and Susswein, 2002). Surprisingly for a behavior that is usually thought of as stereotyped, recent work has revealed great variability in the operation

Received Dec. 9, 2005; revised May 9, 2006; accepted May 23, 2006.

This work was supported by National Institutes of Health Grant R01 NS41497 (V.B.). We thank Dr. Klaudiusz R. Weiss for his generous support.

Correspondence should be addressed to Dr. Vladimir Brezina, Department of Neuroscience, Box 1065, Mount Sinai School of Medicine, 1 Gustave L. Levy Place, New York, NY 10029. E-mail: vladimir.brezina@mssm.edu. DOI:10.1523/JNEUROSCI.5277-05.2006

Copyright $\odot 2006$ Society for Neuroscience $\quad$ 0270-6474/06/267056-15\$15.00/0 of this feeding system. Essentially all parameters of the cycling of the CPG, the bursts of motor neuron firing, contractions of the muscles, and the movements of the behavior are extremely variable from one cycle to the next (Horn et al., 2004; Brezina et al., 2005; Lum et al., 2005; Zhurov et al., 2005b). As we document here, there is great variability also within each cycle, in particular, in the irregular timing of the successive spikes within each motor neuron burst. Intriguingly, Zhurov et al. (2005b) found that these irregular bursts are nevertheless synchronized down to even the individual spike level in the corresponding motor neurons on the two sides of the animal, suggesting that the detailed spike timing may have functional significance.

This, however, is puzzling. Like many other invertebrate muscles (Hoyle, 1983; Morris and Hooper, 1997, 1998; Hooper et al., 1999; Zoccolan et al., 2002), the buccal muscles of Aplysia are thought to be "slow." That is, it would be assumed that they respond only to the overall spike rate, integrated over long times, regardless of the detailed spike timing. Here we work in vitro with one representative buccal muscle, the accessory radula closer (ARC) muscle, and its two motor neurons B15 and B16 (Cohen et al., 1978). By replaying into the motor neurons the spike patterns recorded during normal feeding in vivo and other, comparable patterns with particular statistical properties, we investigate how the B15/B16-ARC neuromuscular transform (Brezina et al., 2000a) transforms the spike patterns into muscle contraction shapes. We find that the transform in fact operates at an interface between "fast" and "slow" regimens. It is fast enough that the 
timing of individual spikes greatly influences the detailed contraction shape. At the same time, slow integration of the spike pattern through the nonlinear transform allows the detailed spike timing (not merely the overall spike rate) to determine also overall parameters of the contraction such as the overall contraction amplitude. Indeed, motor neuron firing lacking the normal variability of spike timing would fail to contract the muscle adequately during feeding behavior.

\section{Materials and Methods}

All new experiments in this paper were done in the standard in vitro preparation used for recording motor neuron-elicited contractions of the ARC muscle (Cohen et al., 1978; Weiss et al., 1979) (for more recent references and use, see Horn et al., 2004; Zhurov et al., 2005a,b). Briefly, the preparation consisted of the bilateral buccal ganglia, the two ARC muscles, and the connecting buccal nerves 3 through which the buccal motor neurons B15 and B16 innervate the ARC muscle. (The cerebral ganglion, connected to the buccal ganglia by the cerebral-buccal connectives, was also retained.) The buccal ganglia (but not the cerebral ganglion) were desheathed. One of the ARC muscles was pinned out in a separate subchamber and connected to an isotonic transducer (model 60-3000; Harvard Apparatus, Holliston, MA) to measure the length of the muscle with a light counterbalancing load. [A few experiments were done simultaneously with both of the ARC muscles, pinned out in separate subchambers and connected to separate transducers (see Fig. 5) (Zhurov et al., 2005b).] The ipsilateral motor neurons B15 and B16 were impaled with standard intracellular microelectrodes and connected to an intracellular amplifier (Axoclamp 2A/B; Molecular Devices, Palo Alto, CA). Under the control of an external stimulator (Grass S48/S88; AstroMed, West Warwick, RI), the motor neurons were stimulated with brief square current pulses to fire spikes in the desired pattern. All signals were sampled and recorded simultaneously by a computer using Digidata 1322A data-acquisition hardware and pClamp 8/9 software (Molecular Devices). When modulators were applied (see Figs. 7-9), they were added to the muscle subchamber so that only the muscle was exposed. Experiments were done at $14-17^{\circ} \mathrm{C}$ or at room temperature $\left(21-24^{\circ} \mathrm{C}\right)$; the results were similar with respect to the phenomena of interest here and have been pooled.

Beyond these standard techniques, only our method of generating the pattern of current pulses injected into the motor neurons (and hence, with one spike per pulse, the pattern of motor neuron spikes) warrants additional comment. When simple regular patterns were required (see Fig. 9), they were generated either by the Grass stimulator itself or the Grass stimulator was controlled by a standard electrophysiological timer (Pulsemaster A300; World Precision Instruments, Sarasota, FL). When irregular patterns were required, however, they were generated by a dedicated arbitrary timer that translated a given, arbitrary list of spike times into a correspondingly timed sequence of transistor-transistor logic (TTL) pulses that then triggered the Grass stimulator. The arbitrary timer was built from a standard IBM-compatible personal computer (Dell Computer Company, Round Rock, TX) running, under Microsoft (Seattle, WA) Windows 2000 or Windows XP Professional, a program custom written in Microsoft Visual C++.NET 7.0, with hardware-control routines from the DataAcq SDK DLL library (Data Translation, Marlboro, MA), that generated the TTL pulses through a DT335 digital input/ output board (Data Translation). Early versions of the program implemented the timing using Windows Timer routines, but, because Windows 2000/XP is not a real-time operating system, these could not guarantee sufficient timing accuracy for some purposes. Later versions of the program therefore used calibrated timing loops in the body of the program itself while setting the process and thread priority to the highest level to freeze all other processes, including those of Windows, for the duration of the timing sequence.

\section{Results}

Is the ARC a slow muscle?

The ARC muscle (or, more properly, the B15/B16-ARC neuromuscular transform) certainly has a number of slow characteris- tics, which can be seen, for example, in Figure 1. Single isolated spikes fired by the ARC motor neurons B15 and B16 elicit no contraction of the muscle at all. Neither do trains of spikes, however long, that are fired at frequencies lower than some threshold frequency, which varies between muscles but is often $4-6 \mathrm{~Hz}$ (Brezina et al., 1997, 2003b) (Fig. 1B). Trains fired at frequencies higher than this begin to contract the muscle only after a considerable delay, usually only after 5 or 10 spikes, hundreds of milliseconds or even seconds after the beginning of the spike train. The contraction then builds slowly, over many seconds. When the spike train ends, the contraction relaxes equally slowly (Brezina et al., 2003b) (Fig. 1A,C). All of this suggests that the contraction integrates the motor neuron spike pattern over relatively long times, over many spikes. It might then be expected that, on faster timescales, the precise temporal arrangement of the spikes should not much matter (Morris et al., 2000; Zoccolan et al., 2002). Provided they have the same average frequency, irregular spike trains should elicit much the same contraction as regular trains, and no single spike in the train should have any great influence on the contraction.

There is, however, the following anecdotal observation. ARC muscle contractions have traditionally been studied in vitro as in Figure 1, with short regular trains or bursts of spikes elicited by intracellular current injection into the cell bodies of the motor neurons, repeated at regular intervals. In general, each identical burst elicits an exactly identical contraction (Horn et al., 2004) (see below). Now and again, however, one contraction can be markedly smaller, sometimes in a distinctly "quantal" manner [Brezina et al. (2003b), their Fig. 6]. A likely reason is that some spikes in that burst have failed to propagate all the way out to the muscle. Perhaps even just a single spike has failed, yet there is a marked change in the contraction. We decided to investigate this phenomenon more formally.

\section{A single extra or missing motor neuron spike dramatically alters ARC muscle contraction}

In Figure $1, A$ and $B$, we used short (2s) regular trains of motor neuron $\mathrm{B} 15$ or B16 spikes. After the initial delay, these trains elicited ARC muscle contractions that continued to build through the rest of the train to a peak just after the end of the train. In the middle of these spike trains, we then inserted one extra spike or, conversely, omitted one spike. As can be seen in the representative example in Figure $1 A$, insertion of an extra spike shortened the delay to the beginning of the contraction and greatly increased the peak contraction amplitude (middle). Omission of a spike had the opposite effects (right). Figure $1 B$ summarizes measurements of the peak contraction amplitude from a number of such experiments with different frequencies of motor neuron firing, with both B15 (left) and B16 (right). The phenomena seen in Figure $1 A$ occurred with both motor neurons, at all frequencies from the smallest that elicited any contraction up to at least $10 \mathrm{~Hz}$. For both motor neurons, there were statistically highly significant differences $(p<0.001)$ (Fig. 1) overall between the control (gray circles), extra-spike (black circles), and missing-spike (open circles) contraction amplitudes.

In Figure $1 C$, we used longer (10 s) trains of motor neuron spikes, which allowed the contraction to build to near steady state before the spike was inserted or omitted. The insertion of a single extra spike then elicited a dramatic transient increase in contraction amplitude (middle), whereas the omission of a single spike elicited a transient decrease (right).

Thus, at least under some circumstances, a single spike does matter. A single spike can have great influence on the contraction. 
More generally, therefore, irregularities in the spike pattern fired by the motor neurons have the potential to shape the contraction to a very significant degree. Next, we show that the muscle does in fact experience such irregular motor neuron spike patterns during the normal operation of the feeding system.

\section{Real spike patterns of the motor} neurons $B 15$ and $B 16$ are irregular

Although the ARC contractions have traditionally been studied with regular spike patterns, it has become increasingly evident that regular spike patterns are not very physiological. As mentioned in Introduction, recent work has revealed great variability in the operation of the Aplysia feeding system at all levels, including that of the motor neuron firing (Horn et al., 2004; Brezina et al., 2005; Lum et al., 2005; Zhurov et al., 2005b). All of the work so far, however, concerned itself only with the variability in the overall parameters of each cycle, such as the mean frequency of the motor neuron spikes fired during the entire burst in each cycle, from one cycle to the next. In the same data, there was clearly also great variability within each cycle, in particular, variability of the timing of the individual motor neuron spikes within the burst. However, this variability was not previously quantified. Here, in Figures 2 and 3 , we reanalyzed the data available from the previous work at that more detailed level. Two datasets in particular provided relevant, complementary information. In the first dataset, the motor neuron spikes were recorded directly with intracellular techniques but in vitro. In the second dataset, the spikes had to be inferred from extracellular electrical activity, but this was done in vivo during normal feeding.

Motor neuron B15 and B16 spike patterns during feeding motor programs in vitro Horn et al. (2004) (see also Zhurov et al., 2005a) recorded the firing of the motor neurons B15 and B16 during feeding motor programs elicited in vitro by electrical stimulation of the esophageal nerve, one of the nerves that in the intact animal carry food-related sensory information to and trigger the activity of the feeding CPG. The motor neuron spike patterns recorded were thus those driven by the endogenous motor programs that in the intact animal would have produced entire, physiological cycles of the feeding behavior.

In these motor programs, the variability or irregularity of the timing of individual spikes was most conspicuous in the firing of motor neuron $\mathrm{B} 16$ during the radula-protraction phase of the program (Horn et al., 2004; Zhurov et al., 2005b). Figure $2 \mathrm{~A}$ shows the spike patterns of $\mathrm{B} 16$ during the protraction phases of four representative programs and plots their instantaneous firing
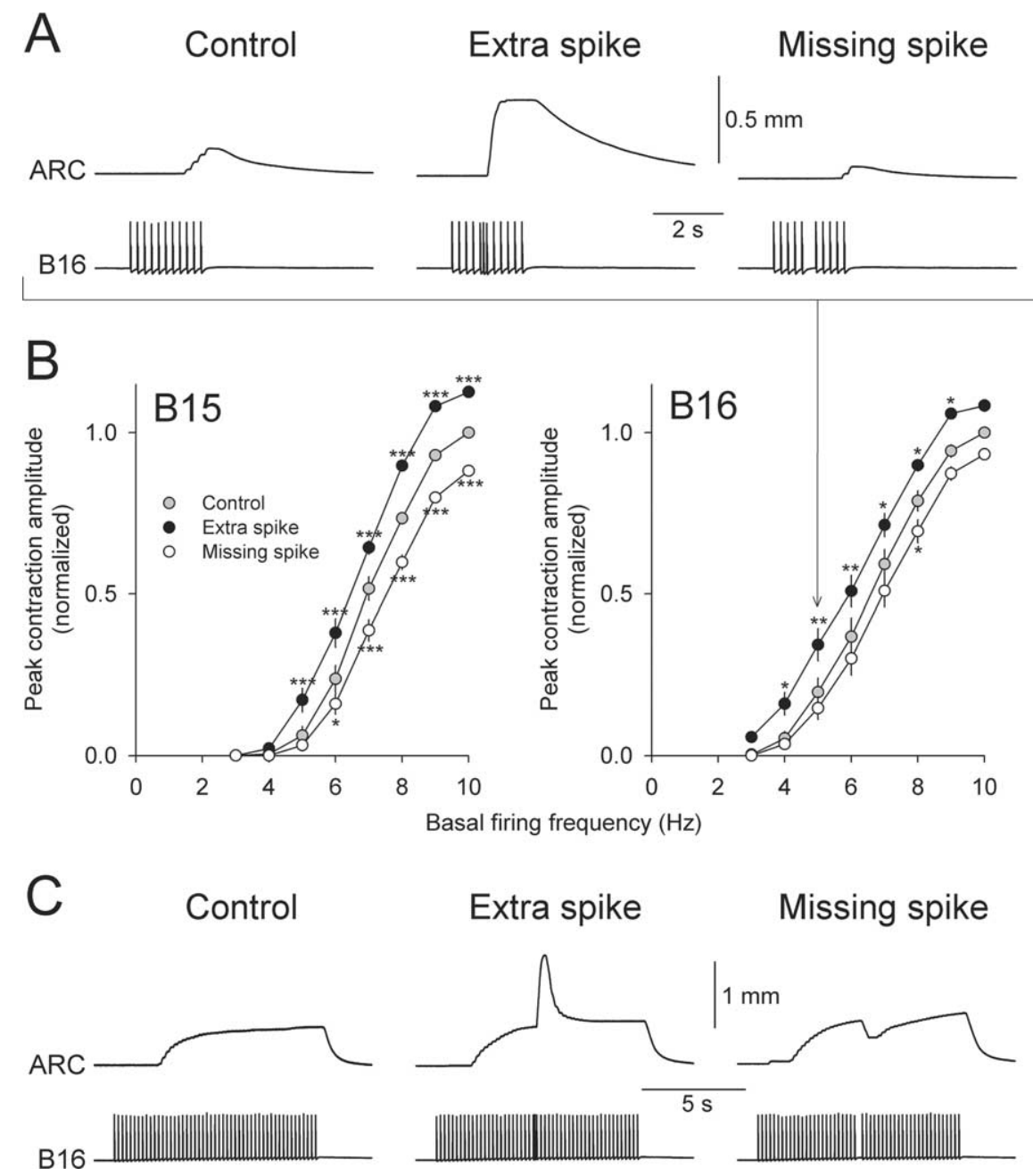

Figure 1. A single extra or missing motor neuron spike dramatically alters ARC muscle contraction. $A$, Representative example with short regular spike trains. Motor neuron B16 was stimulated by intracellular current injection to fire a 2-s-long train at a basal frequency of $5 \mathrm{~Hz}$ (left), which was then modified by the insertion (middle) or omission (right) of a single spike in the middle of the , Group data of measurements of peak contraction amplitude from all experiments like that in $\boldsymbol{A}$. Triplets of control extra-spike, and missing-spike contractions were recorded as in $\boldsymbol{A}$ with different basal firing frequencies (each repeated twice in Statistical significance was tested with two-way ANOVA, followed by pairwise multiple comparisons using the Holm-Sidak test. The overall differences between the control (gray circles), extra-spike (black circle), and missing-spike (open circles) conditions, difference between the extra-spike and control or the missing-spike and control values at a particular frequency. $C$, Representative example with longer regular spike trains. As in $\boldsymbol{A}$ except that motor neuron B16 was fired for $10 \mathrm{~s}$.

frequency functions $\left(f_{\mathrm{B} 16}\right)$. These functions fluctuate greatly, instead of remaining at some steady, mean level as they would in regular firing.

To quantify the variability in the entire dataset, Figure $2 B$ plots the distribution, scaled so as to approximate the probability density function, of all 31,226 B16 interspike intervals measured from the 583 protraction phases in the dataset (see legend). The distribution is broad, containing many intervals that are manyfold different from each other. The 10th, 25th, 50th (median), 75th, and 90th percentiles of the distribution are shown by the thin vertical lines; the coefficient of variation $(\mathrm{CV})$ is 0.68 .

This total variability includes, however, global components that are not relevant for us here, for example, those reflecting the overall differences in mean firing frequency in different programs 
A
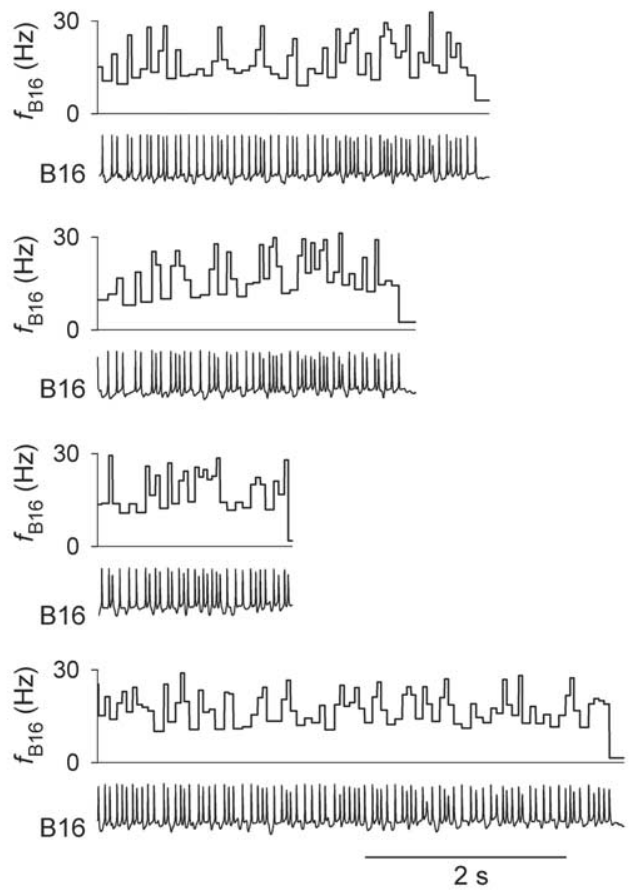

$2 \mathrm{~s}$
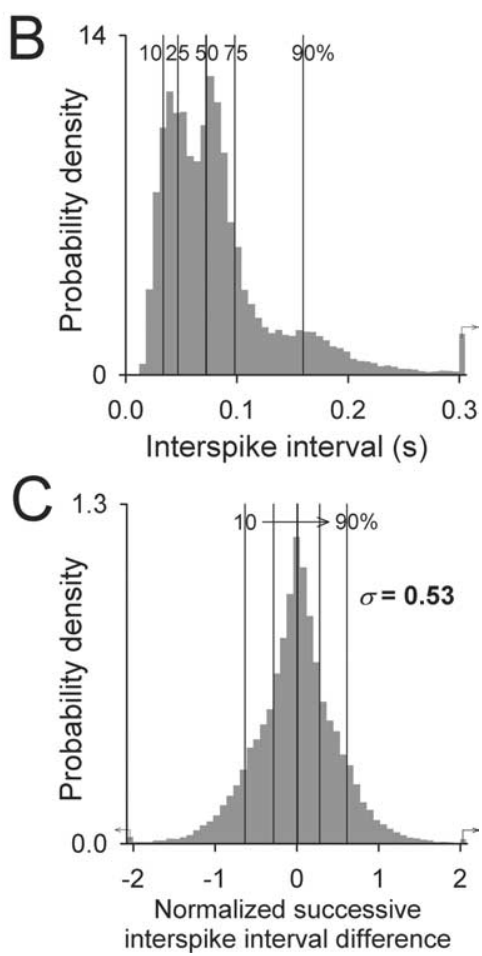

Figure 2. Intraburst variability of motor neuron B16 spike timing during feeding motor programs in vitro. Reanalysis of a dataset of motor programs elicited by electrical stimulation of the esophageal nerve recorded by Horn et al. (2004) (see also Zhurov et al., 2005a). A, Motor neuron B16 spike patterns recorded intracellularly during the radula-protraction phases of four representative programs, with the corresponding instantaneous firing frequency function $\left(f_{\mathrm{B} 16}\right)$, computed by assigning to each time point in an interspike interval the reciprocal of the duration of that interspike interval. $\boldsymbol{B}$, Distribution of all B16 interspike intervals during protraction phases in the entire dataset, subject however to the following selection criterion. Because the B16 firing frequency often gradually increases over the first part of protraction (Brezina et al., 2005; Zhurov et al., 2005a,b), only the second half of each protraction was considered and accepted only if it contained $\geq 30$ interspike intervals. This left from the original dataset a subset of 31,226 interspike intervals from the second halves of 583 protractions from 28 experiments, included in the distribution shown. The distribution is scaled so as to approximate the probability density function. The thin vertical lines mark the 10th, 25th, 50th (median), 75th, and 90th percentiles. $C$, The corresponding distribution of the differences between successive B16 interspike intervals, normalized by the mean interval in each protraction half. Computed from the same data as in $\boldsymbol{B}$, this distribution therefore contains 31,226 - 583 = 30,643 interspike interval differences. The first and last bars contain pooled smaller and larger values, respectively, indicated by the small left- and right-pointing arrows.

or preparations. To extract from the total variability just the local variability of the spike pattern, Figure $2 C$ plots, for the same dataset as in Figure $2 B$, the distribution of the differences between successive interspike intervals, normalized by the mean interval in each protraction phase. With this normalization, +1 and -1 on the abscissa represent, respectively, increases and decreases in interspike interval from one interval to the next that are equal in magnitude to the mean interval. There are many differences as large or larger than this, a very large variability. The SD of the distribution, $\sigma$, which with the normalization is comparable to the $\mathrm{CV}$, is 0.53 .

\section{Motor neuron B15 and B16 spike patterns during spontaneous feeding in vivo}

Horn et al. (2004) also recorded, with chronically implanted wire electrodes, electrical activity in the ARC muscle in intact animals engaged in spontaneous feeding. Electrical activity recorded in the ARC muscle in this way is believed to reflect the large excitatory junctional currents elicited in the muscle by the spikes of the motor neurons B15 and B16 (Cropper et al., 1990a,c). Under favorable circumstances, events attributable to the individual spikes of B15 and B16 can be distinguished, so that the electrical activity can be decomposed into the spike times of the two motor neurons. One representative recording of Horn et al. (2004), of the electrical activity during an entire $\sim 2.5 \mathrm{~h}$ "meal" comprising 749 cycles of the feeding behavior, was decomposed in this way by Brezina et al. (2005). Figure $3 A$ shows the instantaneous firing frequency functions of the two motor neurons, with a schematic representation of the individual spikes underneath, during a representative segment of $13 \mathrm{cy}$ cles from the meal. The two motor neurons fired, in rough synchrony, one burst in each cycle of the behavior. These bursts varied greatly from one cycle to the next, as analyzed by Horn et al. (2004) and Brezina et al. (2005), but also, as can be seen in Figure $3 A$, contained great variability in the timing of their individual spikes.

This is documented in the rest of Figure 3 . Figure $3 B$ shows, in the same way as Figure $2 B$ did for the in vitro dataset, the distributions of all 7847 B15 (left) and 25,116 B16 (right) intraburst interspike intervals measured from all 749 cycles of the meal (see legend). Again, the bulk of each distribution is broad, and there is furthermore a long right-hand tail, extending even offscale, in which the intervals are many times longer than those at the left-hand end of the distribution. The $\mathrm{CV}$ is 0.85 for B15 and 1.05 for B16.

Figure $3 C$ shows, in the same way as did Figure $2 C$, the corresponding distributions of the successive interspike interval differences, normalized by the mean interval. Again, there are many differences as large as the mean interval $(+1$ or -1 on the abscissa) or even twice the mean inter$\operatorname{val}(+2$ or -2$) . \sigma$ is 1.02 for B15 and 1.16 for B16.

Finally, we examined the first return maps, that is, plots of the interval $n+1$ against the interval $n$ (data not shown). These maps had essentially no structure, suggesting that successive interspike intervals were drawn from the distributions in Figure $3 B$ essentially at random, without significant second-order interaction between intervals.

Altogether, the variability of the spike timing within bursts that we found here, with $\mathrm{CV}$ and $\sigma$ values often $\sim 1$, is a very large variability, approximately equal in magnitude to that found previously in overall parameters such as the mean firing frequencies across bursts (Horn et al., 2004; Brezina et al., 2005; Lum et al., 2005; Zhurov et al., 2005b).

\section{Motor neuron spike patterns with different timing produce different muscle contraction shapes}

The results in Figure 1 already suggested that the variable timing of the motor neuron B15 and B16 spikes would pass through the B15/B16-ARC neuromuscular transform to produce different shapes of ARC muscle contraction. To begin to examine this more rigorously, we performed a series of experiments in which we constructed simple patterns of the motor neuron spikes that differed only in their timing. To what extent would the resulting contractions differ? 
A representative experiment from this series is shown in Figure $4 A$, in this case with motor neuron B15. In each experiment, for a given mean frequency of firing $\bar{f}$ (in Fig. $4 A$, it was $7 \mathrm{~Hz}$ ), we constructed six different patterns of 100 spikes each (in Fig. $4 A$, for example, the patterns 1-6 shown schematically at the top). The successive interspike intervals in each pattern were drawn at random from a Gaussian distribution with mean $1 / \bar{f}$ and SD $1 / 4 \bar{f}$. Each pattern thus had the same number of spikes and essentially the same duration, mean spike frequency $\bar{f}$, and other statistical properties, but a different pattern of timing of the spikes. The CV of the interspike intervals in these patterns was $(1 / 4 \bar{f}) /$ $(1 / \bar{f})=0.25$, a degree of variability well within, indeed conservatively smaller, than that found in the real motor neuron spike patterns in the previous section. The motor neuron was fired with each pattern 10 times in succession. In Figure $4 A$, the 10 resulting ARC muscle contraction waveforms (offset from each other by a fixed amount for clarity) are shown down each column. This was repeated with each of the six different patterns, shown in the different columns of Figure $4 A$ (for additional details, see legend of Fig. 4).

Examining the contraction waveforms in Figure $4 A$, three basic features are immediately apparent. First, the complex spike patterns produced complex contraction shapes. Second, repetition of the same pattern, down each column, produced a remarkably similar contraction shape each time. The deviation of each of the 10 contraction waveforms from their mean in each column (Fig. $4 \mathrm{~A}$, bottom, plotted on the same scale as the individual waveforms) was very small. [Furthermore, even the small deviation that was seen in these experiments was generally not random, but rather was correlated across the whole contraction waveform, and tended to progress, perhaps reflecting some process such as slow intrinsic modulation or fatigue of the muscle, systematically through the 10 repetitions (legend of Fig. 4). In other words, the contraction waveform tended to change, usually decrease, in overall size but preserved its detailed shape.] Third, although they had the same spike number and mean frequency, different patterns, across the columns, produced very different contraction shapes.

Figure $4 B$ shows a similar experiment performed with motor neuron B16, with very similar results. The three basic features are apparent also with B16.

To quantify these features, we computed the distance between the contraction waveforms. Specifically, having sampled each of two waveform at $m$ time points, we computed the root mean square difference over the $m$ pairs of values (see equation in legend of Fig. 4). Geometrically, this can be thought of as computing a normalized Euclidean distance between the two waveforms represented as points in the $m$-dimensional space spanned by the $m$
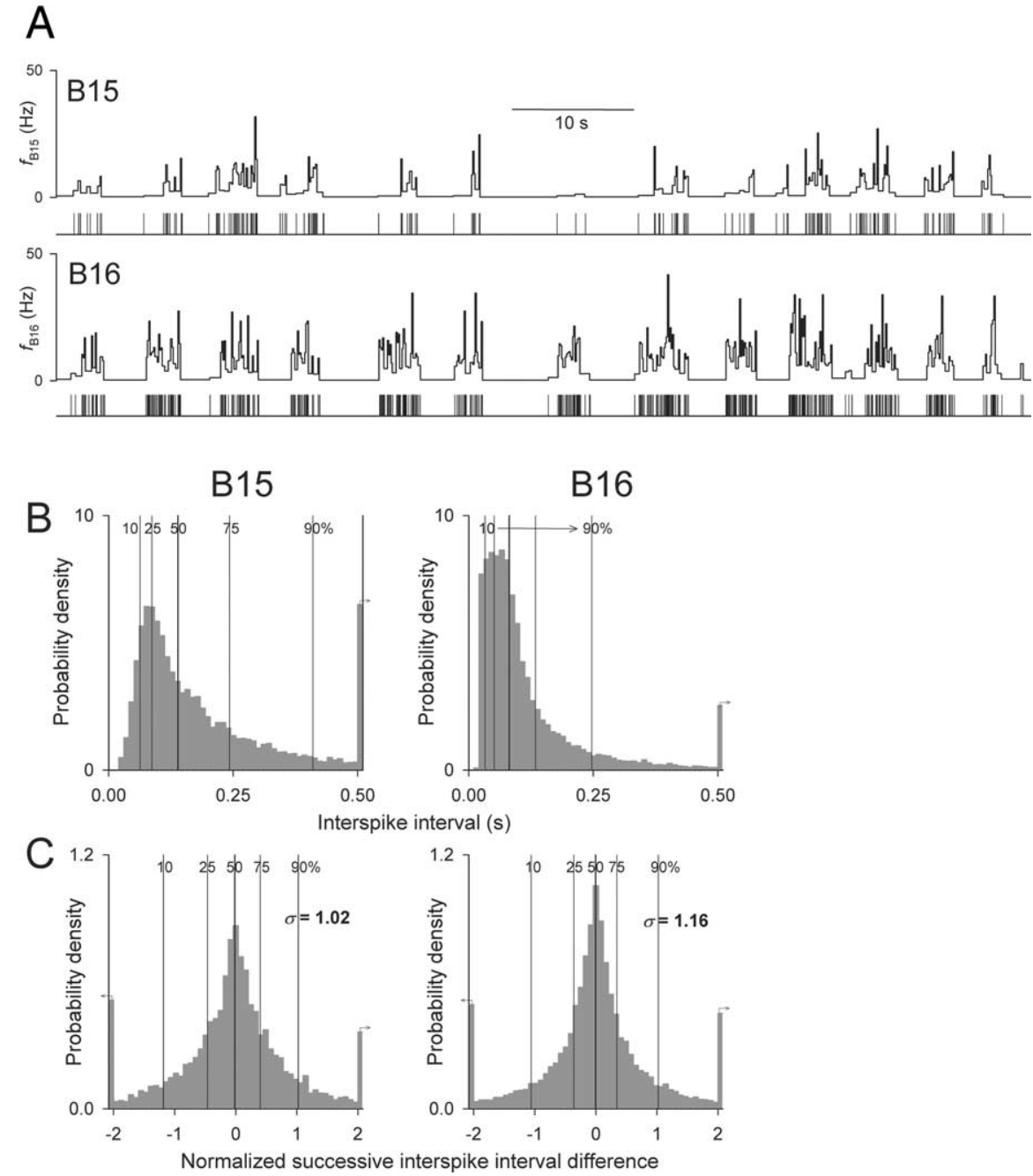

Figure 3. Intraburst variability of motor neuron B15 and B16 spike timing during spontaneous feeding in vivo. Reanalysis of a chronic recording by Horn et al. (2004) of electrical activity in the ARC muscle during an entire $\sim 2.5 \mathrm{~h}$ meal comprising 749 cycles of feeding behavior, then decomposed by Brezina et al. (2005) into the instantaneous firing frequency functions of the motor 列 13 cycles of the instantaneous firing frequency functions with a schematic 25,116 B16 intervals were identified as intraburst intervals, here defined simply as intervals $<1 \mathrm{~s}$. This definition, less restrictive than the complete definition of Brezina et al. (2005), implicitly allowed a somewhat different number of bursts $(722$ for B15, 953 for B16) than cycles (749). B, Distributions of all 7847 B15 (left) and 25,116 B16 (right) intraburst interspike intervals. Plot details as in Figure 2B. C, The corresponding distributions of the 7125 B15 (left) and 24,163 B16 (right) successive intraburst interspike interval differences, normalized by the means of all of the B15 and B16 intraburst interspike intervals. Plot details as in Figure $2 C$.

samples. For example, we sampled each of the contraction waveforms in Figure $4 A$ every 10 ms over the $\sim 14.3$ s duration of the spike pattern. This mapped each of the waveforms to a point in a 1430-dimensional space. Computing the pairwise distances between all of the waveforms in the experiment then allowed us to quantify how similar in fact were the contraction waveforms produced by repetition of the same spike pattern and how different they were from those produced by other patterns. Geometrically, it showed the degree to which each set of similar waveforms was clustered together in the $m$-dimensional space far from the clusters of other, different waveforms.

We computed the distances between the contraction waveforms produced by repetition of the same pattern and those produced by different patterns for each experiment in the entire series. The results are shown in Figure $4 C$, bars 3 and 4 (for motor neuron B15) and 7 and 8 (for motor neuron B16). There were altogether 28 experiments, with mean spike frequencies $\bar{f}$ ranging 
A $\quad$ B15

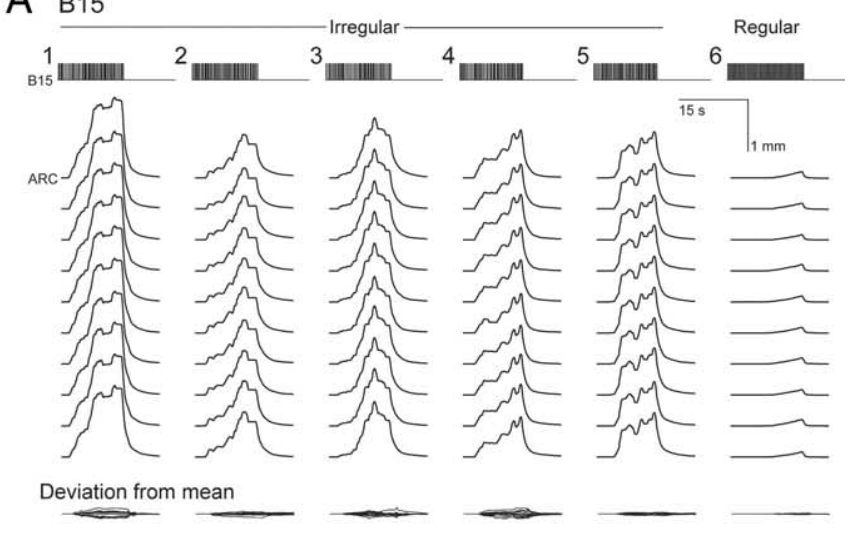

B $\quad$ B16

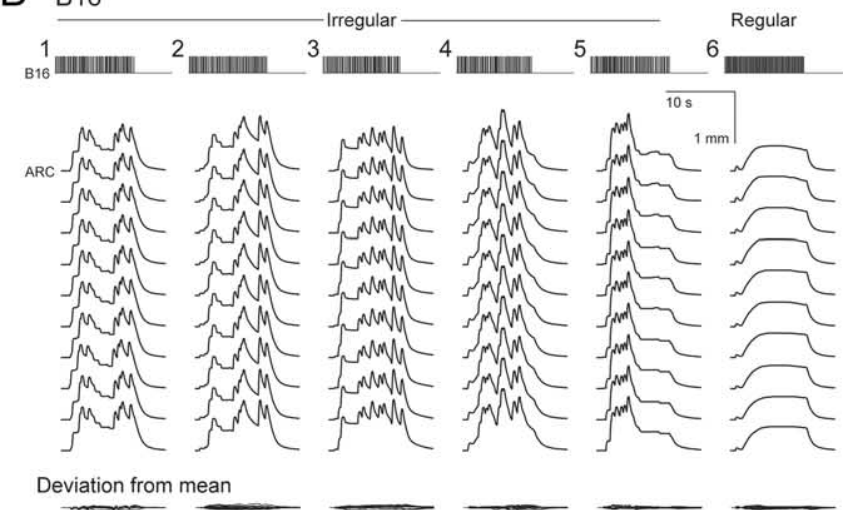

C

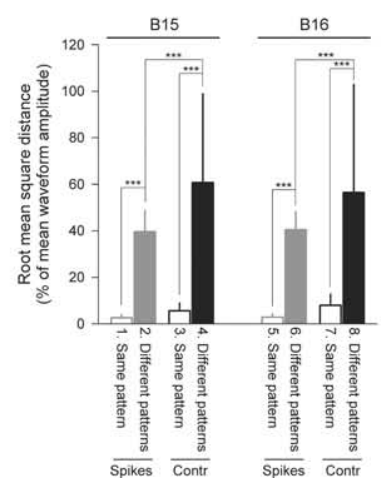

Figure 4. Motor neuron B15 and B16 spike patterns with different timing produce different ARC muscle contraction shapes. $A$, Representative experiment with motor neuron B15. $A$, Top, The six motor neuron B15 spike patterns that were used in the experiment, shown schematically. Each pattern had 100 spikes, a nominal mean spike frequency $\bar{f}=7 \mathrm{~Hz}$, and thus a nominal duration of $14.29 \mathrm{~s}$. The irregular patterns $1-5$ were constructed by drawing the successive interspike intervals from the positive part of a Gaussian distribution with mean $1 / \bar{f}=$ $142.9 \mathrm{~ms}$ and SD $1 /(\overline{4 f})=35.7 \mathrm{~ms}$. The regular pattern 6 was constructed with the same mean interspike interval but $S D=0$ (see Results, Variable motor neuron spike timing maintains contraction amplitude). $A$, Middle, The resulting ARC muscle contraction waveforms. The motor neuron was fired with each spike pattern 11 times in succession, with 1 min rest intervals between the repetitions. Repetition 1 was discarded (see below). The waveforms produced by repetitions $2-11$ are shown (offset from each other by an arbitrary fixed amount for clarity) down each column. After a rest interval of $>1 \mathrm{~min}$, this was repeated with each of the other spike patterns, as shown across the columns. $A$, Bottom, The superimposed deviations (differences) of each of the 10 individual contraction waveforms from the ensemble mean of the waveforms in each column, plotted on the same scale as the individual waveforms. $B$, Representative experiment with motor neuron B16. As in $A$, except with patterns of 100 B 16 spikes at a nominal mean frequency $\bar{f}=9 \mathrm{~Hz}$. C, Group data for distances between contraction waveforms, and the spike patterns that produced them, from the entire series of experiments. All experiments were done as in $\boldsymbol{A}$ and $\boldsymbol{B}$, with six different spike patterns per experiment. However, as noted in Results (Motor neuron spike patterns with different timing produce different from 5 to $17 \mathrm{~Hz}$, with $\mathrm{B} 15$, and 50 experiments, with $\bar{f}$ ranging from 6 to $17 \mathrm{~Hz}$, with B16. As expected, there was a tendency for the distances between the waveforms produced by different patterns to decrease as $\bar{f}$ increased and the absolute timescale on which the patterns differed became shorter. However, this tendency was relatively minor, and all experiments with each motor neuron were pooled (for additional details, see legend of Fig. 4).

In the same way as with the contraction waveforms, we also computed the distances between the spike patterns that produced them. (We used the actual spike patterns recorded as fired by the motor neurons during the experiment rather than the constructed patterns with which the motor neurons were stimulated.) To compute the distances, we first converted the spike patterns into the corresponding continuous waveforms, instantaneous firing frequency functions like those seen in Figures $2 \mathrm{~A}$ and $3 A$. The distances between the spike patterns are shown in Figure $4 C$, bars 1 and 2 (for B15) and 5 and 6 (for B16).

Examining Figure $4 C$, we see the following. (1) The motor neurons B15 and B16 behaved in all respects similarly. (2) The repetitions of the "same" spike pattern (bars 1 and 5) were, as expected, very similar but not exactly identical; the distance between them was very small but not exactly 0 . Presumably, this was because there was some variability or "noise," albeit a very small amount only, in the processes that translated the injected current corresponding to the constructed pattern to the actual spikes. (3) The different spike patterns (bars 2 and 6) differed, in contrast, by $\sim 40 \%$. The difference in mean distance between the repetitions

\section{$\leftarrow$}

muscle contraction shapes), there was some tendency for the contraction waveforms produced by successive repetitions of the same pattern to change, usually to decrease in amplitude. Whenever the peak amplitude of the contraction waveform produced by repetition 11 of a pattern differed by $>20 \%$ from that produced by repetition 2, all repetitions of the pattern were discarded. For the same reason, repetition 1, which followed a longer period of rest and often produced a significantly larger contraction waveform, was routinely discarded. Altogether, this left a dataset of 10 repetitions each of 125 patterns in 28 experiments with motor neuron B15 and 10 repetitions each of 210 patterns in 50 experiments with B16. (The experiments were done with different mean spike frequencies $\bar{f}$, ranging from 5 to $17 \mathrm{~Hz}$ with B15 and 6 to $17 \mathrm{~Hz}$ with B16, but all were pooled here.) The spike pattern actually recorded during each of these repetitions was converted into the instantaneous firing frequency function by assigning to each time point in an interspike interval the reciprocal of the duration of that interspike interval (see Figs. $2 A, 3 A$ ). The instantaneous firing frequency function was then sampled at $m$ time points from the time of the first spike over an interval equal to the duration of the longest pattern in the entire experiment (because the patterns were constructed from random interspike intervals, their durations were not exactly identical), every $10 \mathrm{~ms}$, in $\boldsymbol{A}$ for example for a total of 1430 samples. The contraction waveform produced by the pattern was sampled in the same way. The root mean square distance $d$ between the sampled amplitudes $a$ of two patterns or waveforms 1 and 2 was computed using the following equation:

$$
d=\sqrt{\frac{1}{m} \sum_{i=1}^{m}\left(a_{\text {waveform } 1, i}-a_{\text {waveform } 2, i}\right)^{2}} .
$$

This was done for all pairwise combinations of patterns or waveforms within each experiment. From all of these distances, four mean values were then computed within each experiment: $\bar{d}_{\text {spikes same patrem, }}$ the mean distance between spike patterns that were nominally the same, that is, were repetitions of each other; $\bar{d}_{\text {spikes, different pattern' }}$, the mean distance between patterns that were nominally different; $\bar{d}_{\text {contr, same pattern, }}$ the mean distance between contraction waveforms produced by patterns that were nominally the same; and $\bar{d}_{\text {contr, different pattern, }}$, the mean distance between waveforms produced by patterns that were nominally different. To allow pooling across multiple experiments, these mean distances were further normalized by the mean amplitude of all of the patterns or waveforms in the experiment. Plotted in $C$, finally, are the means $\pm S D$ of the four normalized mean distances from the 28 experiments with motor neuron B15 (bars 1-4) and from the 50 experiments with B16 (bars 5-8). Statistical significance was tested with two-way ANOVA, followed by pairwise multiple comparisons using the Holm-Sidak test; ${ }^{* * *} p<0.001$. 
of the same pattern and the different patterns (i.e., between bars 1 and 2, and bars 5 and 6) was statistically highly significant $(p<0.001)$ (see legend). (4) These distances between the spike patterns were then translated relatively faithfully to the distances between the contraction waveforms that the spike patterns produced. The distances between the contraction waveforms tended to be somewhat larger, but not very much larger, than the distances between the spike patterns. As with the spike patterns themselves, the difference in mean distance between the contraction waveforms produced by the same and different spike patterns (between bars 3 and 4, and bars 7 and 8) was statistically highly significant $(p<0.001)$. (5) With the repetitions of the same spike pattern, the distance between the contraction waveforms was only slightly larger than that between the spike patterns themselves. In this case, the difference in mean distance between the spike patterns and contraction waveforms (between bars 1 and 3, and bars 5 and 7) was not statistically significant $(p>0.05)$. Thus, only a minimal amount of variability or noise was added by the processes that transformed the spike pattern into the contraction waveform. (6) With different spike patterns, in contrast, there was a highly significant difference $(p<0.001)$ in the mean distance between the spike patterns and contraction waveforms (between bars 2 and 4, and bars 6 and 8). In this case, the transformation of the spike patterns to contraction waveforms added a significant amount of variability.

Overall, we can summarize these results thus. When the motor neuron B15 or B16 spike pattern, the input to the B15/B16ARC neuromuscular transform, is the same, so, to a high degree of precision, is the output, the ARC muscle contraction. In other words, the neuromuscular transform is highly deterministic. At the same time, complex spike patterns are transformed into equally complex contraction shapes, and different patterns into different shapes. The transform does not (on the realistic timescales that we examined here) attenuate the variability or irregularity of its input, as we would expect to happen if the ARC were in fact a slow muscle. Instead, the variability appears in the output perhaps even somewhat amplified (result 6 above). One possible mechanism of this amplification will be seen below.

\section{Contraction shapes produced by replaying the real motor neuron spike patterns}

The spike patterns that we used in the previous section had the advantage that they could be constructed to our specifications, but they were artificial. In a complementary series of experiments, therefore, we replayed through the B15/B16-ARC neuromuscular transform the real spike patterns of the motor neurons $\mathrm{B} 15$ and B16, in particular those recorded in vivo during the spontaneous $2.5 \mathrm{~h}$ meal that we reanalyzed in Figure 3.

When replayed several times in succession, these patterns, too, produced very similar contractions each time. However, over the entire $2.5 \mathrm{~h}$ duration of the patterns the tendency of the contractions to gradually change, usually to decrease in overall amplitude, often became quite significant. Therefore, in most experi- ments, we replayed just 20 min segments (or, in some experiments described later, 5 min segments) chosen at random from the $2.5 \mathrm{~h}$ patterns. Then the B15/B16-ARC neuromuscular transform appeared to be as deterministic with the real patterns as it was with the artificial patterns. Rather than documenting this again, in Figure 5, we demonstrate an additional related property. When we replayed the same 20 min segment of the real patterns simultaneously into the left and right motor neurons B15, it produced remarkably similar contraction waveforms of the left and right ARC muscles. The same was seen with the left and right motor neurons B16. Thus, in addition to being highly deterministic, the B15/B16-ARC neuromuscular transform appears to be essentially identical on the two sides of the animal. Previously, Zhurov et al. (2005b) found that, during feeding motor programs in vitro like those reanalyzed here in Figure 2, the firing of the left and right motor neurons $\mathrm{B} 15$, and of the left and right motor neurons B16, is very precisely synchronized by common synaptic input down even to the level of the individual spikes in the pattern. This identity of spike patterns, together with the identity of the neuromuscular transforms, will presumably result in ARC muscle contractions that are identical down even to small details of their shape on the two sides of the animal during feeding behavior.

It can be appreciated already in Figure 5 that the real, irregular motor neuron spike patterns produced a very irregular ARC muscle contraction waveform. Additional examples can be seen in Figures 6 and 7. The burst of motor neuron spikes in each cycle produced a distinct peak of muscle contraction, but in each cycle, this contraction was very different in its overall parameters such as its peak and mean amplitude, as well as in its detailed shape. With the real patterns, unlike with the artificial patterns, the spike burst in each cycle differed not just in its detailed timing but also in its overall parameters such as its spike number, mean frequency $\bar{f}$, and duration. The variability of these overall parameters of the spike pattern from cycle to cycle was clearly a major 

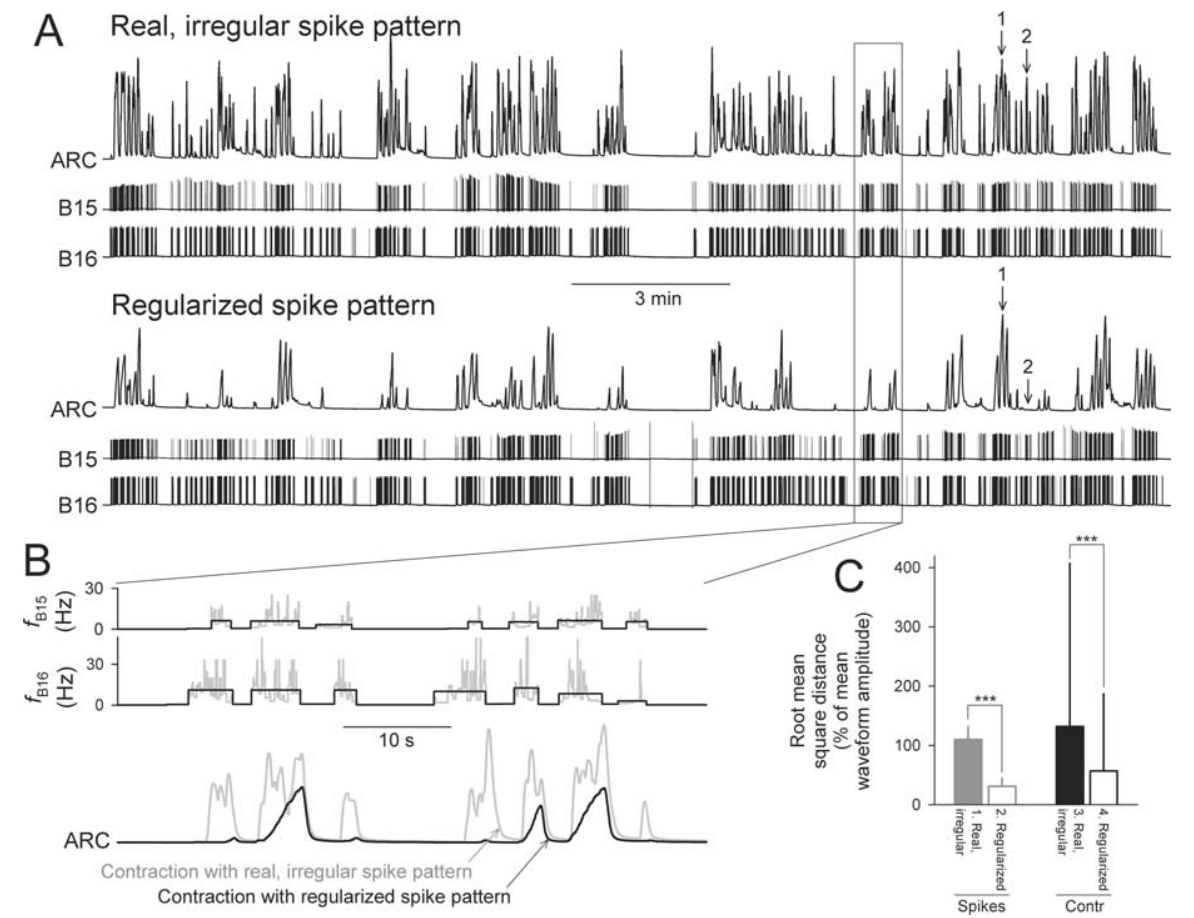

Figure 6. $A R C$ muscle contractions produced by replaying the real, irregular and corresponding regularized motor neuron B15 and B16 spike patterns: representative experiment and analysis of distance between waveforms. $\boldsymbol{A}, \boldsymbol{B}$, Representative experiment. A 20 min segment was chosen at random from the real motor neuron B15 and B16 spike patterns during the spontaneous $2.5 \mathrm{~h}$ meal in Figure 3. Cycles were demarcated using the complete definition of Brezina et al. (2005) whereby each cycle contained one burst each of B15 and B16 spikes, each burst having $\geq 10$ spikes with interspike intervals $<1 \mathrm{~s}$. This particular segment comprised 138 cycles. The corresponding regularized segment was constructed by replacing each real, irregular burst with a burst of the same mean frequency but with all interspike intervals equal. The experiment was then performed as follows. (1) The real, irregular segment was replayed, stimulating both motor neurons B15 and B16 simultaneously with their respective patterns. (2) The regularized segment was replayed similarly. (3) The real, irregular segment was replayed again. A rest interval of $>1$ min was interposed between (1) and (2) and between (2) and (3). (1) was discarded, and (2) and (3) were compared. The result that (2) produced smaller contractions than (3) was therefore conservative with respect to fatigue or other progressive decrease of the contractions during the experiment. $A$, The entire 20 min segment of the real, irregular motor neuron B15 and B16 spike patterns and the resulting $A R C$ muscle contractions (top 3 records) and the corresponding segment of regularized spike patterns and contractions (bottom 3 records). $\boldsymbol{B}$, Expansion of the indicated excerpt from $\boldsymbol{A}$. The spike patterns are shown converted into the real, irregular (gray) and regularized (black) instantaneous firing frequency functions; the contractions are shown superimposed. C, Group data for distances between spike patterns and contraction waveforms from the entire series of experiments. Altogether, the dataset contained 1423 cycles from 18 experiments. Cycles were compared pairwise only within the same experiment and only if their spike patterns were sufficiently similar in the spike numbers and durations (and hence mean frequencies) of their B15 bursts, as well as those of their B16 bursts. The spike numbers were required to differ by no more than four spikes (the SD of the distribution of the spike number per burst over all 1423 cycles was 16.2 spikes, range of $0-150$ spikes) and the burst durations by no more than $0.4 \mathrm{~s}$ (the SD over all cycles was $1.68 \mathrm{~s}$, range of $0.11-20.95 \mathrm{~s}$ ). These criteria allowed 1078 comparisons. For each comparison, the four distances $d_{\text {spikes, real, irregular }}$, between the real, irregular spike patterns; $d_{\text {spikes, regularized }}$, between the corresponding regularized patterns; $d_{\text {contr, real, irregular, }}$, between the contraction waveforms produced by the real, irregular patterns; and $d_{\text {contr, regularized }}$, between the waveforms produced by the regularized patterns, were computed and normalized as in Figure 4C. For the spike patterns, the distances and normalizing mean amplitudes were computed separately for B15 and B16 and averaged. Plotted in the figure are the means \pm SD of the four normalized distances from all 1078 comparisons (bars 1-4). Statistical significance was tested with two-way ANOVA, followed by pairwise multiple comparisons using the Holm-Sidak test; all pairwise differences (only two are indicated by ${ }^{* * *}$ ) were highly significant $(p<0.001)$.

determinant of the variability of the overall parameters of the contraction, as investigated in previous studies (Brezina et al., 1997, 2000a, 2005). The detailed spike timing then presumably determined the detailed shape of the contraction, as with the artificial patterns.

To confirm that the detailed spike timing indeed played that role with the real patterns as with the artificial patterns, we "regularized" the real spike patterns within each burst. A representative experiment is shown in Figure 6, in which the meaning of regularization can be seen by examining the instantaneous firing frequency functions $f_{\mathrm{B} 15}$ and $f_{\mathrm{B} 16}$ in the excerpt expanded in Fig- ure $6 B$. In replaying the spike patterns into the motor neurons, we replaced each irregular burst with a regular burst that had the same overall parameters (the same spike number, mean frequency $\bar{f}$, and duration) as the irregular burst, but whose interspike intervals were all equal so that throughout the burst the motor neuron fired tonically at $\bar{f}$. The overall parameters of the bursts still varied from cycle to cycle as before, and this variability presumably made the same contribution as before to the variability of the overall parameters of the contractions from cycle to cycle. Only the variability of spike timing within each burst, and its contribution, was eliminated. In each experiment, we compared the contraction waveform produced by the real, irregular patterns with that produced by the regularized patterns. Both motor neurons B15 and B16 were fired simultaneously with their respective real, irregular or regularized patterns (for details, see legend of Fig. 6). The entire 20 min contraction waveforms from the representative experiment can be seen in Figure 6A, and expanded excerpts are superimposed in Figure $6 B$ (bottom).

It is clear in Figure 6 that the contraction waveforms produced by the real, irregular patterns (Fig. 6B, bottom, black record) and the regularized patterns (gray record) were indeed very different. To quantify this difference, we computed root mean square distances between the waveforms, and the patterns that produced them, as we did with the artificial patterns. Specifically, we computed the distances between pairs of cycles. A complication was that no two cycles of the real, irregular spike patterns had exactly the same overall parameters. We therefore computed the pairwise distances between cycles whose overall parameters were similar within certain criteria (see legend of Fig. 6).

The results for 1078 cycle pairs from 18 experiments are shown in Figure 6C. We can see the following. (1) All distances were larger than they were with the artificial patterns in Figure 4C. This presumably reflected the fact that the cycles being compared were not exactly identical in their overall parameters. If they had been, the regularized spike patterns of each pair should have differed by $\sim 3 \%$ as in Figure $4 C$ rather than $\sim 30 \%$ (Fig. $6 C$, bar 2). (2) Apart from this, however, the distances in Figure $6 C$ exhibited the same features as those in Figure 4C. In particular, (3) the distances between the real, irregular spike patterns were substantially larger than those between the corresponding regularized patterns. This difference (between bars 1 and 2) was statistically highly significant $(p<0.001)$. (4) This difference then translated into a statistically highly significant difference between the contraction waveforms produced by the real, irregular and the regularized patterns (between bars 3 
and 4). (5) For both the real, irregular and the regularized patterns, the distances between the contraction waveforms were larger than those between the spike patterns. In both cases, the difference (between bars 1 and 3, and bars 2 and 4) was statistically highly significant (not indicated in Fig. 6C; see legend).

We concluded that, as with the artificial patterns, the variability of detailed spike timing that actually occurs in the real patterns fired by the motor neurons during normal feeding does indeed pass, unattenuated and perhaps even somewhat amplified, through the B15/B16-ARC neuromuscular transform to produce variability in the detailed shape of the muscle contraction.

\section{Variable motor neuron spike timing maintains contraction amplitude}

Interestingly, it appeared that the detailed spike timing played a major role in determining not just the detailed contraction shape but also the overall parameters of the contraction. This was already suggested by an observation that we made with the artificial patterns during the experiments described above. Although included in our analysis just like any other pattern, one of the six patterns in each of those experiments was in fact a regularized pattern (Fig. 4A,B, Regular), with the same mean frequency $\bar{f}$ as the other five irregular patterns but with all interspike intervals equal. As can be seen in Figure 4, $A$ and $B$, these regular patterns often produced contractions that were considerably smaller than those produced by the irregular patterns. Not only was the peak contraction amplitude smaller, as was to be expected, but so was the mean contraction amplitude and the total "amount" of contraction produced by the pattern. The difference between the contractions produced by the regular and irregular patterns was more pronounced (for reasons that will become clear below) at smaller values of $\bar{f}$. To quantify the difference, we computed, from each experiment in our dataset with $\bar{f} \leq 10 \mathrm{~Hz}$, the ratio of the mean contraction amplitude produced by the regular pattern to that produced by the irregular patterns. Over all of the experiments, this ratio was $0.65 \pm 0.31$ (mean $\pm \mathrm{SD} ; n=16$ ) for motor neuron $\mathrm{B} 15$ and $0.63 \pm 0.48(n=13)$ for motor neuron B16. In other words, the regular patterns produced on average only $\sim 65 \%$, and in some experiments (such as that in Fig. 4A) much less, of the contraction that the irregular patterns produced, although the regular and irregular patterns had all the same overall parameters and differed only in their spike timing. Paired $t$ tests showed that the difference between the mean contraction amplitudes produced by the regular and irregular patterns was statistically significant ( $p<0.01$ for B15; $p<0.05$ for B16).

The same was true with the contractions produced by the real, irregular patterns and the corresponding regularized patterns. In Figure 6, for instance, it is clear that the regularized contractions (Fig. $6 B$, bottom, black record) were considerably smaller than the real, irregular contractions (gray record). It was not the case, however, that the contraction was simply scaled down by the

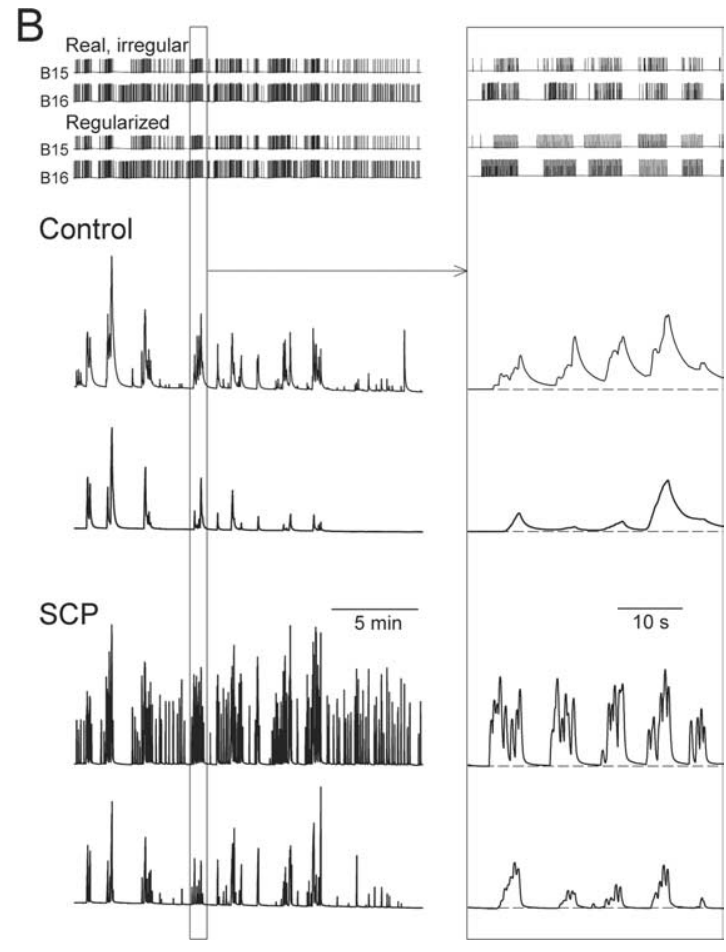

Figure 7. $A R C$ muscle contractions produced by replaying the real, irregular and regularized motor neuron B15 and B16 spike patterns: representative effects of modulators. $A$, Effect of buccalin. A 5 min segment was chosen at random from the real motor Bottom, The resulting contractions in the presence of buccalin. $\boldsymbol{B}$, Effect of SCP. As in $\boldsymbol{A}$, except with a 20 min segment of the spike patterns and $100 \mathrm{nM} S C P_{B}$. The records at right expand the indicated portion of the main records.

same amount in each cycle. Rather, in some cycles (Fig. 6A, cycle $1)$, the contraction appeared to be almost unchanged in mean and sometimes even peak amplitude, whereas in other cycles (cycle 2), it was completely eliminated. Over all 138 cycles of the segment in Figure 6, the regularized mean contraction amplitude was only $\sim 39 \%$ of the real, irregular amplitude.

Group data for this phenomenon will be seen below. First, we show how the phenomenon is modified by the state of modulation of the B15/B16-ARC neuromuscular transform.

\section{Neuromodulators sharpen the effect of variable spike timing on contraction amplitude}

The B15/B16-ARC neuromuscular system is heavily modulated by numerous neuromodulators that alter in various ways the relationship between the firing of the motor neurons and the contractions of the muscle, that is, the neuromuscular transform (for review, see Hooper et al., 1999; Brezina et al., 2000b, 2003a). The core of the modulation is "intrinsic" (Cropper et al., 1987a; Katz and Frost, 1996): the motor neurons themselves, when they fire, release several families of modulatory neuropeptide cotransmitters. Here we tested two of the principal neuropeptides whose effects are relatively straightforward and well understood (see below), namely small cardioactive peptide $\mathrm{B}\left(\mathrm{SCP}_{\mathrm{B}}\right)$, released from motor neuron B15 (Lloyd et al., 1984; Cropper et al., 1987a, 1990c; Whim and Lloyd, 1990; Vilim et al., 1996), and buccalin A, the principal form of the buccalin family of neuropeptides released from both motor neurons B15 and B16 (Cropper et al., 1988, 1990b; Vilim et al., 1994, 1996, 2000).

Figure 7, $A$ and $B$, shows two representative experiments in 


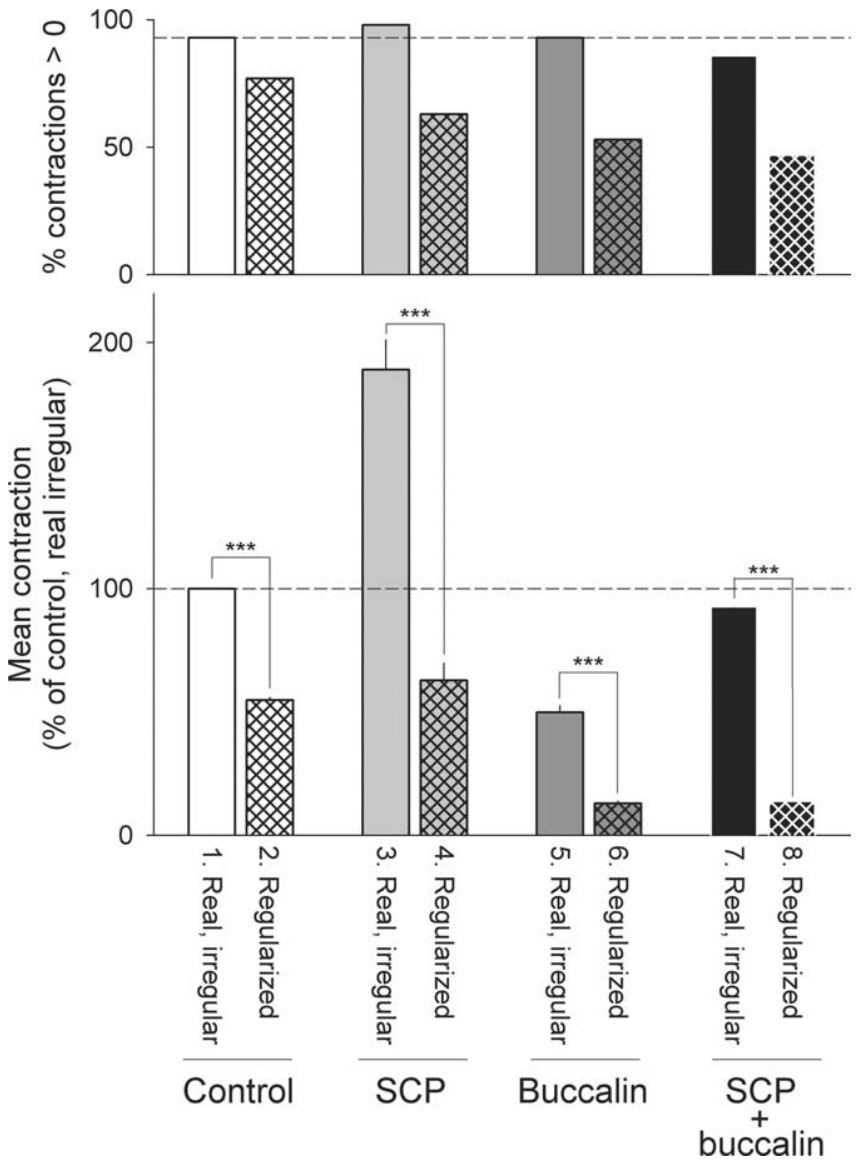

Figure 8. In the real motor neuron B15 and B16 spike patterns, variable spike timing maintains ARC muscle contraction amplitude: group data for the effects of spike pattern regularization and modulators. Experiments were performed as in Figure 7. Altogether, the dataset contained 1423 cycles from 18 experiments under control conditions (same as analyzed in Fig. 6C), 793 cycles from 8 experiments in the presence of $100 \mathrm{nM}, 1 \mu \mathrm{M}$, or $10 \mu \mathrm{M} \mathrm{SCP}$ (the different concentrations had qualitatively similar effects and were pooled), 492 cycles from 9 experiments in the presence of $100 \mathrm{~nm}$ or $1 \mu \mathrm{m}$ buccalin $\mathrm{A}$, and 818 cycles from 8 experiments in the presence of both $S C P_{B}$ and buccalin $A$. In each cycle, contraction amplitude was measured from the first B15 or B16 spike in that cycle to the first spike in the subsequent cycle; the baseline contraction amplitude at the first spike in that cycle was subtracted (Brezina et al., 2005). Top, Percentage of the cycles with above-zero contraction amplitude. Bottom, Mean \pm SE of the mean contraction amplitude in each cycle normalized by the mean amplitude of the corresponding real, irregular contraction under control conditions. Cycles in which the latter was 0 were excluded. This was acceptable because contractions were eliminated but generally not newly created under the other conditions except with SCP, where the exclusion probably underestimated the total amount of contraction that was produced by the spike pattern. For the mean contraction amplitudes, statistical significance was tested with two-way ANOVA, followed by pairwise multiple comparisons using the Holm-Sidak test. The overall difference between the real, irregular and the regularized amplitudes was highly significant ( $p<0.001$ ), as was that difference under each of the individual modulator conditions ( $\left.{ }^{* * *} p<0.001\right)$. All other individual differences were also highly significant $(p<0.001)$ except those between bars 1 and $7(p<0.05)$ and between bars 2 and 4 , and bars 6 and $8(p>0.05)$.

which we again replayed segments of the real, irregular spike patterns from the $2.5 \mathrm{~h}$ meal and the corresponding regularized patterns (top two pairs of records) and compared the resulting contraction waveforms. We did this first under control conditions (middle pair of records) and then in the presence of buccalin or SCP (bottom pair of records). As can be seen in Figure 7A, buccalin generally decreased the amplitude of the contractions. However, its effect was distinctly greater on the regularized contractions than on the real, irregular contractions so that the difference between the two was enhanced. SCP, in contrast, gener- ally increased the amplitude of the contractions (Fig. $7 B$ ). However, it had a greater effect on the real, irregular contractions than on the regularized contractions so that, again, the difference between the two was enhanced.

Group data, measured from 1423 cycles from 18 experiments, are shown in Figure 8. At the top is plotted the percentage of cycles with measurable contraction, and at the bottom is plotted the (normalized) mean contraction amplitude in those cycles (for details, see legend). The solid bars show these measurements for the real, irregular contractions, and the cross-hatched bars show these measurements for the corresponding regularized contractions, under control conditions (bars 1 and 2) and in the presence of SCP (bars 3 and 4), buccalin (bars 5 and 6), and finally both SCP and buccalin (bars 7 and 8).

Examining Figure 8, we see the following. (1) Under all four conditions, the mean amplitude of the regularized contractions was considerably smaller than that of the real, irregular contractions. The difference in each case (between bars 1 and 2, bars 3 and 4, bars 5 and 6 , and bars 7 and 8 ) was statistically highly significant $(p<0.001)$. When applied alone, (2) SCP increased and (3) buccalin decreased the mean amplitude of the real, irregular contractions. In both cases, the effect (the difference between bars 1 and 3, and bars 1 and 5) was statistically highly significant (not indicated in Fig. 8; see legend). (4) Together, SCP and buccalin gave an intermediate mean amplitude of the real, irregular contractions that was not very different from that under control conditions (compare bars 1, 7). (5) SCP, buccalin, and especially SCP and buccalin together modulated the real, irregular and the regularized contractions in a quantitatively differential manner, enhancing the difference between their mean amplitudes. Under control conditions, the mean amplitude of the regularized contractions was $55 \%$ of that of the real, irregular contractions; in the presence of SCP it was 33\%, in the presence of buccalin it was $26 \%$, and in the presence of both SCP and buccalin it was only $15 \%$ (ratios of bar 2 to bar 1, bar 4 to bar 3, bar 6 to bar 5, and bar 8 to bar 7 , respectively). Statistical analysis (one-way ANOVA) of these ratios computed on a cycle-by-cycle basis showed that the difference between the mean amplitudes of the real, irregular and the regularized contractions was significantly larger $(p<0.001)$ in the presence of SCP or in the presence of buccalin than under control conditions and in the presence of both SCP and buccalin than in the presence of either modulator alone.

Thus, the contractions produced by the regularized spike patterns systematically fail to achieve the amplitude of the contractions produced by the real, irregular patterns. Somehow, the intraburst variability of spike timing (the only feature of the real patterns that the regularized patterns lack) not only shapes the contractions in detail but maintains their overall amplitude. Furthermore, this effect of spike timing variability is sharpened by the endogenous modulators of the system.

\section{Mechanism of amplitude maintenance}

In a final series of experiments, we elicited contractions with short regular trains of spikes, as in Figure 1, under control conditions and in the presence of SCP, buccalin, and both SCP and buccalin. Figure $9 A$ shows a representative experiment. Figure $9 B$ then summarizes measurements of the peak contraction amplitude from a number of such experiments with different frequencies of motor neuron firing, with both B15 (left) and B16 (right).

\section{Control conditions (Fig. 9B, white circles)}

These experiments again revealed a distinct threshold frequency, in this set of experiments $\sim 5 \mathrm{~Hz}$ with both motor neurons, for any contraction at all to be elicited. Given this strong nonlinearity 
in the firing frequency-contraction amplitude relationship, the mechanism of amplitude maintenance could readily be understood as an "iceberg effect" (Volgushev and Eysel, 2000), a form of "pattern dependence” (Brezina et al., 1997). Recall that in our experiments with the artificial spike patterns, the difference in contraction amplitude produced by the irregular and regular patterns was especially pronounced when the mean spike frequency $\bar{f}$ was low, not much higher than the threshold frequency. Similarly, in many of the cycles of the real $2.5 \mathrm{~h}$ meal, motor neuron $\mathrm{B} 15, \mathrm{~B} 16$, or both fired with $\bar{f}$ not much higher, and often indeed lower, than the threshold frequency. [Over all 749 cycles, the median $\bar{f}$ for $\mathrm{B} 15$ was $3.4 \mathrm{~Hz} ; 68.1 \%$ of the cycles had $\bar{f}<5 \mathrm{~Hz}$. For B16, the me$\operatorname{dian} \bar{f}$ was $8.5 \mathrm{~Hz} ; 9.4 \%$ of the cycles had $\bar{f}$ $<5 \mathrm{~Hz}$ (Brezina et al., 2005, their Fig. 11).] In all of these cases, the pattern dependence was consequently strong. In the extreme case (Fig. 6B, last cycle in the excerpt), if $\bar{f}$ was actually lower than the threshold frequency, the regular firing at $\bar{f}$ produced no contraction at all. The corresponding irregular firing, however, contained some intervals at which the instantaneous spike frequency rose above the threshold and produced contractions, the peaks of the iceberg rising above the surface. Even when $\bar{f}$ was somewhat higher than the threshold frequency so that the regular firing at $\bar{f}$ produced some contraction, nevertheless, provided that the range of instantaneous frequencies contained in the irregular firing spanned the nonlinearity in the frequency-contraction relationship, the irregular firing produced a greater peak as well as mean contraction amplitude than the regular firing (Brezina et al., 1997).

This pattern dependence would have been found even if the spike pattern had lasted long enough for the contraction waveform produced by the regular firing, and the mean of that produced by the irregular firing, to reach a steady state. However, the pattern dependence was presumably further enhanced by kinetic factors that were brought to prominence by the fact that the spike bursts were generally short, so that all of these phenomena were taking place as the contraction waveform was still building up. Even when $\bar{f}$ was higher than the threshold frequency so that the regular firing at $\bar{f}$ would eventually have produced a contraction, the delay to the beginning of the contraction was so long that in some cases the contraction did not appear before the spike burst ended. [The median burst duration in the $2.5 \mathrm{~h}$ meal was $3.3 \mathrm{~s}$ (Brezina et al., 2005, their Fig. 11).] Even when the contraction did appear, it appeared late in the burst and rose slowly (Figs. $1 A$, control, 5 , pattern 6 , and $6 B$, black record). However, because the delay to ARC muscle contraction decreases and the rate of contraction increases steeply with increasing motor neuron firing frequency (Brezina et al., 2003b, their Fig. 1 A,C), the intervals of higher instantaneous frequency in the irregular firing sharply accelerated the development of the contraction (compare Figs. $1 A$, middle, 5 , patterns $1-5$, and $9 B$, gray record). Then, once the contraction had developed, it relaxed slowly, and thus promoted the summation of additional contractions, because the ARC muscle relaxation rate decreases with contraction amplitude [Brezina et al. (2003b), their Fig. 1D].
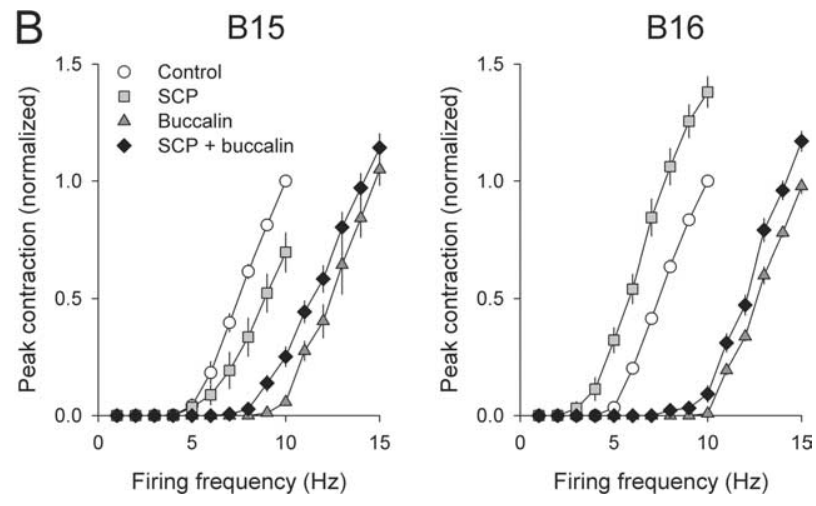

Figure 9. Mechanism of contraction amplitude maintenance and its sharpening by the modulators. $A$, Representative experment. Motor neuron B16 was stimulated to fire for $2 \mathrm{~s}$ at $10 \mathrm{~Hz}$ (bottom) every $60 \mathrm{~s}$, and the resulting ARC muscle contraction contraction amplitude were normalized by the peak amplitude of the control contraction elicited by the same motor neuron firing at $10 \mathrm{~Hz}$. Shown are means \pm SE from 6 control, 8 SCP, 3 buccalin, and 12 SCP plus buccalin experiments with motor neuron B15 (left), and 4 control, 4 SCP, 4 buccalin, and 8 SCP plus buccalin experiments with motor neuron B16 (right). Statistical significance was tested with two-way ANOVA. For both B15 and B16, the four frequency-amplitude relationships were all significantly different from one another $(p<0.01)$.

Finally, another factor along similar lines may have operated more systematically. Unlike with the artificial spike patterns constructed in Figure 4, the real spike bursts during the $2.5 \mathrm{~h}$ meal did not have each interspike interval drawn completely randomly from the distributions in Figure 3B. Rather, there was a modest but systematic tendency for the intervals to decrease, for the spikes to accelerate, toward the end of each burst (Brezina et al., 2005; Zhurov et al., 2005a,b). This would have tended to increase the likelihood that, with the irregular but not the regular firing, the contraction rose above threshold toward the end of each burst.

Effect of buccalin (Fig. 9B, gray triangles)

Buccalin is well known to depress ARC muscle contraction amplitude (Cropper et al., 1988, 1990b; Vilim et al., 1994). The experiments in Figure 9 revealed that buccalin accomplishes this depression by shifting the threshold for contraction to substantially higher frequencies, to $\sim 10 \mathrm{~Hz}$ in this set of experiments. The effect was statistically highly significant (see legend of Fig. 9). As with other modulators of ARC muscle contractions (Orekhova et al., 2003), there was an associated change, in this case an increase, in the delay to contraction (Fig. 9A). Along the lines laid out above, these effects very satisfactorily explained the enhancement by buccalin of the difference between the contractions produced by the regular and irregular firing that we found in Figures 7 and 8 .

\section{Effect of SCP (Fig. 9B, gray squares)}

SCP is well known to potentiate the amplitude of ARC muscle contractions and at the same time to accelerate their relaxation rate (Lloyd et al., 1984; Cropper et al., 1987a, 1990c; Whim and Lloyd, 1990). In some of the experiments in Figure 9, SCP had the opposite effect to buccalin, shifting the contraction threshold to lower frequencies (Fig. 9B, right). However, this effect of SCP was much smaller than that of buccalin and, in any case, would have diminished the difference between the contractions produced by the regular and irregular firing, contrary to what we found in 


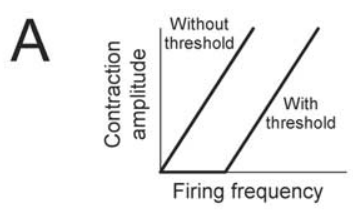

1. Spike pattern

B Without threshold

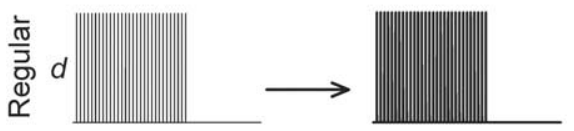

C With threshold
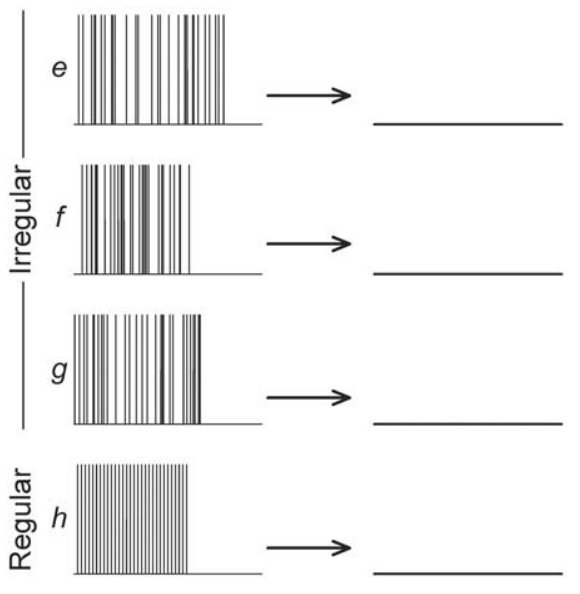

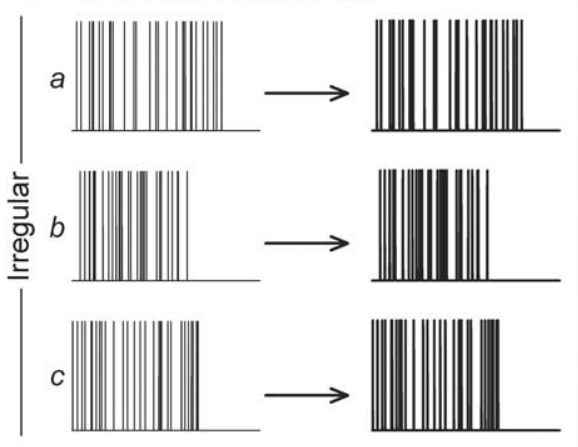

Slow

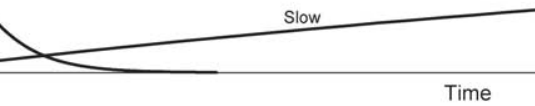

3. Intermediate transform

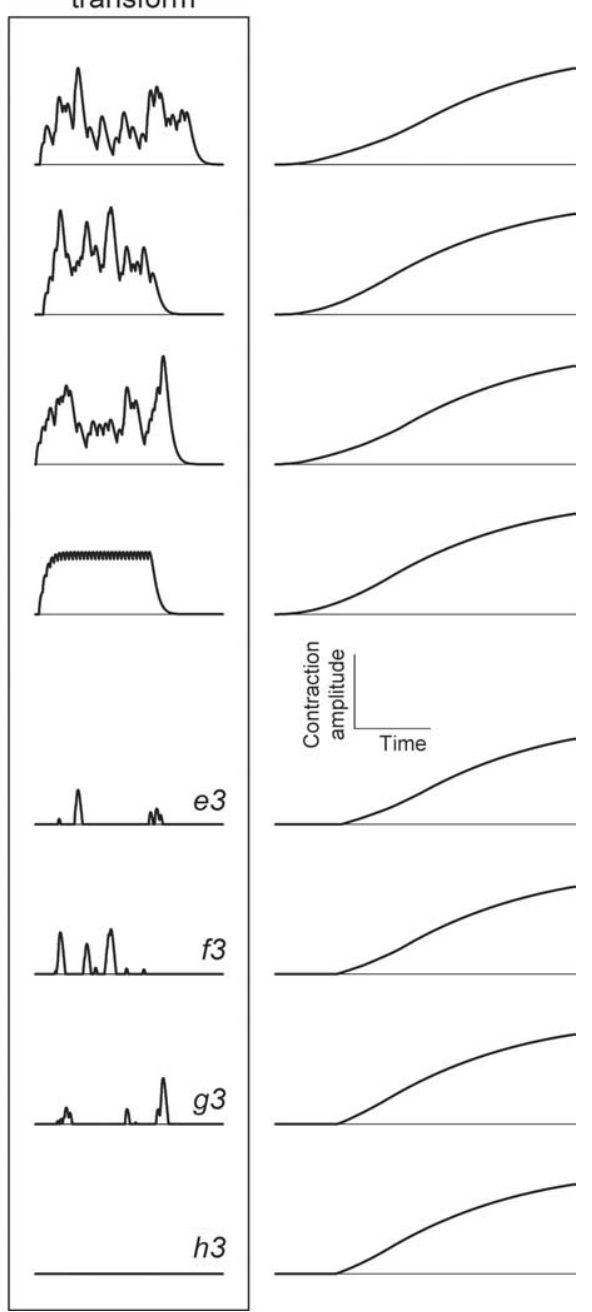

Figure 10. The results found in this paper are explained by a nonlinear neuromuscular transform operating at the interface between fast and slow regimens. Illustration with a minimal model in which the contraction amplitude $c$ at time $t$ is given by

$$
c(t)=\sum_{i=1, t_{i}<t}^{n} a\left(\frac{t-t_{i}}{\tau}\right) \exp \left(1-\left(\frac{t-t_{i}}{\tau}\right)\right),
$$

as the sum of a series of elementary contraction kernels, identical $\alpha$ functions (Dayan and Abbott, 2001) with time constant $\tau$, elicited by $n$ spikes occurring at times $t_{i}=\left\{t_{1}, t_{2}, \ldots, t_{n}\right\}$. $A$, Right shows the elementary kernels with $\tau=0.001$ (fast), 0.1 (intermediate), and 10 (slow; only partly visible) (arbitrary units). Column 1 in $\boldsymbol{B}$ and $\boldsymbol{C}$ shows three irregular spike patterns ( $\boldsymbol{a}-\boldsymbol{C}$ in $\boldsymbol{B}$, repeated as $\boldsymbol{e}-\boldsymbol{g}$ in $\boldsymbol{C}$ ), all with $n=30$ spikes at a mean frequency $\bar{f}=10$ (arbitrary units), constructed by drawing the successive interspike intervals from the positive part of a Gaussian distribution with mean and SD $1 / \bar{f}$. The corresponding regular pattern is also shown $(\boldsymbol{d}, \boldsymbol{h})$. Columns $2-4$ in $\boldsymbol{B}$ and $\boldsymbol{C}$ then show the contraction waveforms $\boldsymbol{c}(t)$ produced by these spike patterns with $\tau=0.001$ (fast, column 2), 0.1 (intermediate, column 3), and 10 (slow, column 4; only partly visible). In this construction, the peak amplitude factor $a$ in the above equation was set to $1 /(e \tau)$, so that the elementary kernels all had a contraction area of 1 , and the contraction waveforms therefore all had the same area, regardless of $\tau$. However, to make them all equally visible, the three elementary kernels in $\boldsymbol{A}$ (right) and the columns $2-4$ of $\boldsymbol{B}$ and $\boldsymbol{C}$ are shown on different vertical scales. In $\boldsymbol{B}$, no threshold was applied; in $\boldsymbol{C}$, the contraction waveform $c(t)$ was passed through a piecewise linear threshold function with the threshold amplitude set to $3 /($ e $\tau)$, i.e., three times the peak amplitude of the elementary kernel. The threshold function is shown schematically in $\boldsymbol{A}$ (left).
Figures 7 and 8 . SCP most likely acted by a different mechanism, modifying not so much the static threshold but rather the kinetic factors noted above. The acceleration of the relaxation rate by SCP (which is very evident in Fig. 9A), together with an acceleration of the contraction rate than underlies or accompanies the potentiation of contraction amplitude by SCP (Brezina et al., 2000b), amounts to a general acceleration of the B15/B16-ARC neuromuscular transform by SCP. As can be seen, for example, in the excerpts expanded in Figure $7 B$ (right), this made the contractions generally faster, less integrative, and more responsive to the instantaneous motor neuron firing frequency. The differential impact of the intervals of high instantaneous frequency, relative to the regular firing at the low, mean frequency, was thereby enhanced.

Effect of both buccalin and SCP (Fig. 9B, black diamonds)

When both modulators were present, the effects of both were expressed simultaneously, apparently in a simple additive manner. Thus, buccalin and SCP together shifted the contraction threshold to higher frequencies much as buccalin did alone (Fig. 9B) and accelerated the relaxation rate of the contractions much as SCP did alone (Fig. 9A, compare the "control," "SCP," and "SCP + buccalin relaxation phase scaled to control" records). The simultaneous expression of the two complementary effects explained, in a very reasonable manner, how both modulators together were able to enhance the difference between the contractions produced by the regular and irregular firing to a significantly greater degree than either modulator did alone (Fig. 8).

\section{Requisite properties of the neuromuscular transform}

In Figure 10, we sought to develop a minimal model of the neuromuscular transform that would reproduce the main experimental results of this paper. In particular, we sought to explain how the intraburst variability of spike timing results in dramatically different detailed contraction shapes (Fig. 4) but at the same time also determines overall properties of the contraction such as the overall contraction amplitude (Figs. 6-8). What properties must the transform have so that these results can both be true at the same time?

First, the transform must be neither too fast nor too slow, but intermediate in speed, relative to the timescale of the input 
spike pattern. Figure $10 B$ shows, in column 1 , three irregular spike patterns $(a-c)$ and the corresponding regular pattern $(d)$. These patterns are passed through a model transform that outputs simply the linear sum of identical elementary contraction kernels elicited by each spike (for details, see legend). The kernels, and so the transform as a whole, are modeled as fast, intermediate, or slow, differing by two orders of magnitude to make the consequences very clear. The individual kernels are shown in Figure $10 \mathrm{~A}$ (right), and the summed contraction waveforms produced by the spike patterns are shown in columns 2 (fast), 3 (intermediate), and 4 (slow) of Figure $10 \mathrm{~B}$. Clearly, the fast transform (column 2) allows the spike pattern to penetrate entirely through to the contraction: the contraction shape simply mirrors the spike pattern. The slow transform, conversely (column 4), integrates the spike pattern so much that none of the pattern penetrates: all patterns produce the same contraction shape. Whether a transform is fast or slow, indeed, is defined simply by these consequences. Only the transform operating at intermediate speed relative to the spike pattern (column 3 ) allows both consequences to be expressed to an appreciable extent. It allows the spike pattern to penetrate sufficiently so that different patterns produce different contraction shapes, yet already integrates sufficiently between spikes so that the elementary kernels overlap and summate to build shapes that are dramatically different from each other, as we saw experimentally in Figure $4, A$ and $B$.

Because, thus far, the transform is linear, all of the contraction waveforms in Figure 10 B, whether produced by irregular or regular patterns, have the same area. The second property that the transform must have to reproduce our results is nonlinearity. Figure $10 C$ repeats Figure $10 \mathrm{~B}$ but passes the contraction waveforms through a threshold nonlinearity, schematically shown in Figure $10 \mathrm{~A}$ (left), that is similar to that found experimentally (Fig. 9B). Now, provided the amplitude of the contraction waveform spans the nonlinearity (for a detailed analysis of the requirements of the interaction, see Brezina et al., 1997), different spike patterns produce different amounts of contraction. In particular, irregular patterns produce contractions that rise above the threshold (Fig. 10C, e3, f3, g3) when the corresponding regular pattern produces no contraction at all $(h 3)$. Note, however, that this happens only when the transform is of the intermediate speed. With fast or slow transforms, the threshold affects all contraction waveforms, whether produced by irregular or regular patterns, equally.

The model transform in Figure 10 qualitatively reproduces our results even without incorporating additional dynamic nonlinearities, such as those attributable to classical synaptic facilitation (Cohen et al., 1978) or the release of the intrinsic modulators from the motor neurons, both of which increase whenever the instantaneous spike frequency increases, that sharpen these effects in the real B15/B16-ARC transform (see Discussion).

\section{Discussion}

\section{Single spike timing matters}

We documented here that, during feeding motor programs in vitro and more importantly during spontaneous feeding in vivo, the motor neurons B15 and B16 fire in bursts with very variable or irregular timing of the individual spikes. With the input of these real spike patterns, the B15/B16-ARC neuromuscular transform does not behave in a simple fast or slow manner but rather operates at the interface between the fast and slow regimens, and it expresses a threshold nonlinearity. As a consequence, the large variability in the timing of the motor neuron spikes is expressed in the contractions of the muscle, indeed, in two ways at once: it not only shapes the detailed contraction at any moment, but, more unexpectedly, it also plays a major role in maintaining the overall integrated amplitude of the contraction. In fact, without the spike timing variability, it appears that there would be no robust contractions of the ARC muscle at all during normal feeding.

The speed and the nonlinearity of the B15/B16-ARC transform were already known before this work (Brezina et al., 2003b), but their functional significance was not fully appreciated. The ARC appeared to be simply a slow muscle. In large part, this was because, like most other muscles, the ARC had only been studied with regular spike patterns. This work therefore joins the growing list of cases (Burke et al., 1976; Zajac and Young, 1980; Markram and Tsodyks, 1996; Abbott et al., 1997; Lisman, 1997; Phares et al., 2003) in which unsuspected phenomena have been revealed when systems have been probed with more realistic, irregular spike patterns and underscores the importance of using such patterns.

\section{Tuning by neuromodulators}

The degree to which the spike timing variability maintains overall contraction amplitude appears to be tuned by endogenous modulators of the B15/B16-ARC system. Here we examined two of the principal modulators, buccalin and SCP. Both sharpen the effect but by different complementary mechanisms. Buccalin raises the contraction threshold, whereas SCP speeds up the neuromuscular transform. Judging by the already known similarities of their actions, other modulators can be predicted to have similar, although perhaps more complex, effects. Thus, serotonin (Weiss et al., 1978; Hooper et al., 1999) is likely to act like SCP, whereas the myomodulins (Cropper et al., 1987b; Brezina et al., 1995; Orekhova et al., 2003) are likely to have both SCP- and buccalin-like effects.

Most of this modulation is intrinsic: the modulators are automatically released from the motor neurons B15 and B16 themselves as they fire. SCP is released from B15, the myomodulins from B16, and the buccalins from both motor neurons (Vilim et al., 1996, 2000). As the motor neurons fire in different patterns, the changing mix of the released modulators, through their multiple effects, dynamically modifies the shape of the ARC muscle contractions. (The B15/B16-ARC neuromuscular transform is therefore deterministic, strictly, only with respect to a particular state of the modulation.) Within this complex network of context-dependent, dynamic interactions, it has been possible to appreciate the functional roles of particular modulatory effects only with the aid of quantitative mathematical modeling (Brezina et al., 2003a,b, 2005). Thus, although some plausible ideas have already emerged here, we expect that the effects that we found here will need to be added to the model before their true functional significance can be understood in the context of the entire interacting physiological system.

\section{Other systems}

Formally, neuromuscular integration in most invertebrate as well as vertebrate systems can usefully be understood in terms much like those of our model in Figure 10. That is, elementary contraction responses to each motor neuron spike summate through a nonlinear neuromuscular transform, often one that rises above a distinct threshold (Hoyle, 1983; Rome and Lindstedt, 1997). What integration occurs then becomes a question of the formal properties that we emphasized here, of the degree of nonlinearity and especially the speed of the transform relative to the timescale and variability of the input spike pattern. These properties are 
such in many systems that it is likely that the phenomena that we described will be quite common.

In Aplysia, many other muscles, not only in the buccal mass but also, for example, in the gill musculature, resemble the ARC in the speed and nonlinearity of their neuromuscular transforms (Carew et al., 1974; Evans et al., 1996; Fox and Lloyd, 1997; Yu et al., 1999), the irregular firing of their motor neurons (Byrne et al., 1978; Morton and Chiel, 1993; Wu et al., 1994; Jing et al., 2004), and their complex modulation (Church et al., 1993; Fox and Lloyd, 1997; Evans et al., 1999; Keating and Lloyd, 1999; Hurwitz et al., 2000). With the real, irregular motor neuron spike patterns, the contractions of these muscles have shapes very much like those that we recorded here in Figures 4-7 (Wu et al., 1994).

The requisite properties also appear to be present in a variety of other invertebrate muscles [the slow muscles of the crustacean stomatogastric system studied by Hooper and colleagues (Morris and Hooper, 1997, 1998; Morris et al., 2000; Thuma et al., 2003) are good candidates] and in slow vertebrate muscles such as various smooth muscles (Hoyle, 1983; Hooper et al., 1999). Even vertebrate skeletal muscles may exhibit similar phenomena. Although their neuromuscular transforms are much faster, so are the spike patterns of their motor neurons (Rome and Lindstedt, 1997). The same phenomena may then simply occur on a faster timescale.

\section{Functional significance in Aplysia feeding behavior}

In the other slow muscles just mentioned, the slow aspect of their character has been particularly emphasized because it is perceived to be part of the solution to a general biological problem. Namely, how can the stochastic variability or "noise" that inevitably arises in any biological system be suppressed to produce a deterministic, controllable output of the system? At multiple levels in the neuromuscular system, this is understood to happen by averaging, both spatial averaging across populations of elements in parallel and temporal averaging in any one element (Wu et al., 1994; Arisi et al., 2001; Zoccolan et al., 2002). Slow muscles will participate by low-pass filtering the variability of their input, the irregularity of the timing of their motor neuron spikes, to produce a smooth, reproducible contraction shape, as in column 4 of Figure 10, $B$ and $C$ (Hoover et al., 2002; Zoccolan et al., 2002; Thuma et al., 2003).

It is quite possible that, at some levels, averaging does take place in the B15/B16-ARC system. For example, temporal and spatial averaging across multiple fibers within the muscle may help produce the deterministic contraction of the whole muscle, as analyzed in lobster muscle by Hoover et al. (2002). In many ways, however, the B15/B16-ARC system appears to have a character that is almost the opposite of that of other neuromuscular systems. In the leech, for instance, Zoccolan et al. (2002) have described how, despite variable motor neuron spike trains and a noisy, nondeterministic neuromuscular transform, averaging across a population of the motor neurons and filtering by the low-pass properties of the muscle nevertheless gives a deterministic motor response. In the Aplysia B15/B16-ARC system, in contrast, the neuromuscular transform is deterministic, there are not many motor neurons across which to average, and, as we have shown here, there is relatively little low-pass filtering by the muscle, so that the variable motor neuron spike trains reliably pass through the transform to produce a motor response that is, in large part, stochastic.

We believe that this is so because, in this case, a stochastic motor response is adaptive. Aplysia must feed in a variable and changing environment about which they do not have very precise information: they do not know, for instance, precisely what movements of the feeding apparatus will most efficiently ingest the next piece of seaweed. We proposed that they nevertheless feed effectively by adopting a trial-and-error strategy in which the feeding CPG generates variability that diversifies the motor neuron spike patterns, muscle contractions, feeding movements, and ultimately the performance of the feeding behavior. At least some cycles of the behavior will succeed, and these cycles can then be repeated and stabilized (Horn et al., 2004; Brezina et al., 2005; Lum et al., 2005; Zhurov et al., 2005b). In the previous work, we studied this variable strategy on the level of whole cycles. Here, we extended our work down to the level of individual spikes. In some ways, the timing of the motor neuron B15 and B16 spikes is well controlled: spatially across the two sides of the animal, the spikes are well synchronized (Zhurov et al., 2005b) and so, with a deterministic neuromuscular transform, are the ARC muscle contraction shapes (Fig. 5). The timing of successive spikes is very variable, however, and, as we have now shown, this greatly diversifies the shapes of the contractions. It appears therefore that the variable feeding strategy is actively implemented even at the level of the individual spikes within each feeding cycle.

\section{References}

Abbott LF, Varela JA, Sen K, Nelson SB (1997) Synaptic depression and cortical gain control. Science 275:221-224.

Arisi I, Zoccolan D, Torre V (2001) Distributed motor pattern underlying whole-body shortening in the medicinal leech. J Neurophysiol 86:2475-2488.

Brezina V, Bank B, Cropper EC, Rosen S, Vilim FS, Kupfermann I, Weiss KR (1995) Nine members of the myomodulin family of peptide cotransmitters at the B16-ARC neuromuscular junction of Aplysia. J Neurophysiol 74:54-72.

Brezina V, Orekhova IV, Weiss KR (1997) Control of time-dependent biological processes by temporally patterned input. Proc Natl Acad Sci USA 94:10444-10449.

Brezina V, Orekhova IV, Weiss KR (2000a) The neuromuscular transform: the dynamic, nonlinear link between motor neuron firing patterns and muscle contraction in rhythmic behaviors. J Neurophysiol 83:207-231.

Brezina V, Orekhova IV, Weiss KR (2000b) Optimization of rhythmic behaviors by modulation of the neuromuscular transform. J Neurophysiol $83: 260-279$.

Brezina V, Orekhova IV, Weiss KR (2003a) Neuromuscular modulation in Aplysia. I. Dynamic model. J Neurophysiol 90:2592-2612.

Brezina V, Orekhova IV, Weiss KR (2003b) Neuromuscular modulation in Aplysia. II. Modulation of the neuromuscular transform in behavior. J Neurophysiol 90:2613-2628.

Brezina V, Horn CC, Weiss KR (2005) Modeling neuromuscular modulation in Aplysia. III. Interaction of central motor commands and peripheral modulatory state for optimal behavior. J Neurophysiol 93:1523-1556.

Burke RE, Rudomin P, Zajac FE (1976) The effect of activation history on tension production by individual muscle units. Brain Res 109:515-529.

Byrne JH, Castellucci VF, Kandel ER (1978) Contribution of individual mechanoreceptor sensory neurons to defensive gill-withdrawal reflex in Aplysia. J Neurophysiol 41:418-431.

Carew TJ, Pinsker H, Rubinson K, Kandel ER (1974) Physiological and biochemical properties of neuromuscular transmission between identified motoneurons and gill muscle in Aplysia. J Neurophysiol 37:1020-1040.

Church PJ, Whim MD, Lloyd PE (1993) Modulation of neuromuscular transmission by conventional and peptide transmitters released from excitatory and inhibitory motor neurons in Aplysia. J Neurosci 13:2790-2800.

Cohen JL, Weiss KR, Kupfermann I (1978) Motor control of buccal muscles in Aplysia. J Neurophysiol 41:157-180.

Cropper EC, Lloyd PE, Reed W, Tenenbaum R, Kupfermann I, Weiss KR (1987a) Multiple neuropeptides in cholinergic motor neurons of Aplysia: evidence for modulation intrinsic to the motor circuit. Proc Natl Acad Sci USA 84:3486-3490.

Cropper EC, Tenenbaum R, Kolks MAG, Kupfermann I, Weiss KR (1987b) 
Myomodulin: a bioactive neuropeptide present in an identified cholinergic buccal motor neuron of Aplysia. Proc Natl Acad Sci USA 84:5483-5486.

Cropper EC, Miller MW, Tenenbaum R, Kolks MAG, Kupfermann I, Weiss KR (1988) Structure and action of buccalin: a modulatory neuropeptide localized to an identified small cardioactive peptide-containing cholinergic motor neuron of Aplysia californica. Proc Natl Acad Sci USA 85:6177-6181.

Cropper EC, Kupfermann I, Weiss KR (1990a) Differential firing patterns of the peptide-containing cholinergic motor neurons B15 and B16 during feeding behavior in Aplysia. Brain Res 522:176-179.

Cropper EC, Miller MW, Vilim FS, Tenenbaum R, Kupfermann I, Weiss KR (1990b) Buccalin is present in the cholinergic motor neuron B16 of Aplysia and it depresses accessory radula closer muscle contractions evoked by stimulation of B16. Brain Res 512:175-179.

Cropper EC, Price D, Tenenbaum R, Kupfermann I, Weiss KR (1990c) Release of peptide cotransmitters from a cholinergic motor neuron under physiological conditions. Proc Natl Acad Sci USA 87:933-937.

Dayan P, Abbott LF (2001) Theoretical neuroscience. Cambridge, MA: MIT.

deCharms RC, Zador A (2000) Neural representation and the cortical code. Annu Rev Neurosci 23:613-647.

Eggermont JJ (1998) Is there a neural code? Neurosci Biobehav Rev 22:355-370.

Elliott CJH, Susswein AJ (2002) Comparative neuroethology of feeding control in molluscs. J Exp Biol 205:877-896.

Evans CG, Rosen S, Kupfermann I, Weiss KR, Cropper EC (1996) Characterization of a radula opener neuromuscular system in Aplysia. J Neurophysiol 76:1267-1281.

Evans CG, Vilim FS, Harish O, Kupfermann I, Weiss KR, Cropper EC (1999) Modulation of radula opener muscles in Aplysia. J Neurophysiol 82:1339-1351.

Fox LE, Lloyd PE (1997) Serotonin and the small cardioactive peptides differentially modulate two motor neurons that innervate the same muscle fibers in Aplysia. J Neurosci 17:6064-6074.

Hooper SL, Brezina V, Cropper EC, Weiss KR (1999) Flexibility of muscle control by modulation of muscle properties. In: Beyond neurotransmission: neuromodulation and its importance for information processing (Katz PS, ed), pp 241-274. Oxford: Oxford UP.

Hoover NJ, Weaver AL, Harness PI, Hooper SL (2002) Combinatorial and cross-fiber averaging transform muscle electrical responses with a large stochastic component into deterministic contractions. J Neurosci 22:1895-1904.

Horn CC, Zhurov Y, Orekhova IV, Proekt A, Kupfermann I, Weiss KR, Brezina V (2004) Cycle-to-cycle variability of neuromuscular activity in Aplysia feeding behavior. J Neurophysiol 92:157-180.

Hoyle G (1983) Muscles and their neural control. New York: Wiley.

Hurwitz I, Cropper EC, Vilim FS, Alexeeva V, Susswein AJ, Kupfermann I, Weiss KR (2000) Serotonergic and peptidergic modulation of the buccal mass protractor muscle (I2) in Aplysia. J Neurophysiol 84:2810-2820.

Jing J, Cropper EC, Hurwitz I, Weiss KR (2004) The construction of movement with behavior-specific and behavior-independent modules. J Neurosci 24:6315-6325.

Katz PS, Frost WN (1996) Intrinsic neuromodulation: altering neuronal circuits from within. Trends Neurosci 19:54-61.

Keating C, Lloyd PE (1999) Differential modulation of motor neurons that innervate the same muscle but use different excitatory transmitters in Aplysia. J Neurophysiol 82:1759-1767.

König P, Engel AK, Singer W (1996) Integrator or coincidence detector? The role of the cortical neuron revisited. Trends Neurosci 19:130-137.

Kupfermann I (1974) Feeding behavior in Aplysia: a simple system for the study of motivation. Behav Biol 10:1-26.

Lisman JE (1997) Bursts as a unit of neural information: making unreliable synapses reliable. Trends Neurosci 20:38-43.

Lloyd PE, Kupfermann I, Weiss KR (1984) Evidence for parallel actions of a molluscan neuropeptide and serotonin in mediating arousal in Aplysia. Proc Natl Acad Sci USA 81:2934-2937.

Lum CS, Zhurov Y, Cropper EC, Weiss KR, Brezina V (2005) Variability of swallowing performance in intact, freely feeding Aplysia. J Neurophysiol 94:2427-2446.

Markram H, Tsodyks M (1996) Redistribution of synaptic efficacy between neocortical pyramidal neurons. Nature 382:807-810.
Morris LG, Hooper SL (1997) Muscle response to changing neuronal input in the lobster (Panulirus interruptus) stomatogastric system: spike number- versus spike frequency-dependent domains. J Neurosci 17:5956-5971.

Morris LG, Hooper SL (1998) Muscle response to changing neuronal input in the lobster (Panulirus interruptus) stomatogastric system: slow muscle properties can transform rhythmic input into tonic output. J Neurosci 18:3433-3442.

Morris LG, Thuma JB, Hooper SL (2000) Muscles express motor patterns of non-innervating neural networks by filtering broad-band input. Nat Neurosci 3:245-250.

Morton DW, Chiel HJ (1993) The timing of activity in motor neurons that produce radula movements distinguishes ingestion from rejection in Aplysia. J Comp Physiol A Neuroethol Sens Neural Behav Physiol 173:519-536.

Orekhova IV, Alexeeva V, Church PJ, Weiss KR, Brezina V (2003) Multiple presynaptic and postsynaptic sites of inhibitory modulation by myomodulin at ARC neuromuscular junctions of Aplysia. J Neurophysiol 89:1488-1502.

Phares GA, Antzoulatos EG, Baxter DA, Byrne JH (2003) Burst-induced synaptic depression and its modulation contribute to information transfer at Aplysia sensorimotor synapses: empirical and computational analyses. J Neurosci 23:8392-8401.

Rome LC, Lindstedt SL (1997) Mechanical and metabolic design of the muscular system in vertebrates. In: Handbook of physiology, Sec 13, Comparative physiology (Dantzler WH, ed), pp 1587-1651. New York: Oxford UP.

Thuma JB, Morris LG, Weaver AL, Hooper SL (2003) Lobster (Panulirus interruptus) pyloric muscles express the motor patterns of three neural networks, only one of which innervates the muscles. J Neurosci 23:8911-8920.

Vilim FS, Cropper EC, Rosen SC, Tenenbaum R, Kupfermann I, Weiss KR (1994) Structure, localization, and action of buccalin B: a bioactive peptide from Aplysia. Peptides 15:959-969.

Vilim FS, Price DA, Lesser W, Kupfermann I, Weiss KR (1996) Costorage and corelease of modulatory peptide cotransmitters with partially antagonistic actions on the accessory radula closer muscle of Aplysia californica. J Neurosci 16:8092-8104.

Vilim FS, Cropper EC, Price DA, Kupfermann I, Weiss KR (2000) Peptide cotransmitter release from motor neuron B16 in Aplysia californica: costorage, corelease, and functional implications. J Neurosci 20:2036-2042.

Volgushev M, Eysel UT (2000) Noise makes sense in neuronal computing. Science 290:1968-1972.

Weiss KR, Cohen JL, Kupfermann I (1978) Modulatory control of buccal musculature by a serotonergic neuron (metacerebral cell) in Aplysia. J Neurophysiol 41:181-203.

Weiss KR, Mandelbaum DE, Schonberg M, Kupfermann I (1979) Modulation of buccal muscle contractility by serotonergic metacerebral cells in Aplysia: evidence for a role of cyclic adenosine monophosphate. J Neurophysiol 42:791-803.

Whim MD, Lloyd PE (1990) Neuropeptide cotransmitters released from an identified cholinergic motor neuron modulate neuromuscular efficacy in Aplysia. J Neurosci 10:3313-3322.

Wu J-Y, Tsau Y, Hopp H-P, Cohen LB, Tang AC, Falk CX (1994) Consistency in nervous systems: trial-to-trial and animal-to-animal variations in the responses to repeated applications of a sensory stimulus in Aplysia. J Neurosci 14:1366-1384.

Yu S-N, Crago PE, Chiel HJ (1999) Biomechanical properties and a kinetic simulation model of the smooth muscle I2 in the buccal mass of Aplysia. Biol Cybern 81:505-513.

Zajac FE, Young JL (1980) Properties of stimulus trains producing maximum tension-time area per pulse from single motor units in medial gastrocnemius muscle of the cat. J Neurophysiol 43:1206-1220.

Zhurov Y, Proekt A, Weiss KR, Brezina V (2005a) Changes of internal state are expressed in coherent shifts of neuromuscular activity in Aplysia feeding behavior. J Neurosci 25:1268-1280.

Zhurov Y, Weiss KR, Brezina V (2005b) Tight or loose coupling between components of the feeding neuromusculature of Aplysia? J Neurophysiol 94:531-549.

Zoccolan D, Pinato G, Torre V (2002) Highly variable spike trains underlie reproducible sensorimotor responses in the medicinal leech. J Neurosci 22:10790-10800. 